\title{
Two new pioneer communities of Sorbus aucuparia and Sorbus aria in the southern Julian Alps
}

\section{Igor Dakskobler ${ }^{1}$}

Key words: phytosociology, syndynamics, successional stage, Sorbus aucuparia, Sorbus aria, Natura 2000, Julian Alps, western Slovenia.

Ključne besede: fitocenologija, sindinamika, sukcesijski stadij, Sorbus aucuparia, Sorbus aria, Natura 2000, Julijske Alpe, zahodna Slovenija.
Received: 28. 4. 2015

Revision received: 15.10 .2015

Accepted: 18. 10. 2015

\begin{abstract}
In the southern Julian Alps we described two communities whose tree layer is dominated by species from the genus Sorbus and noted two successional stages in the overgrowing of abandoned agricultural land (pastures, hay meadows). In the secondary succession on former subalpine pastures above the alp Planina Razor und under the Breginjski Stol ridge, where potential natural vegetation consists of subalpine beech forest, dwarf pine has been overgrown with mountain ash (Sorbus aucuparia) whose stands are classified into the new association Rhododendro hirsuti-Sorbetum aucupariae. Whitebeam (Sorbus aria) has established itself on steep former hay meadows in the belt of altimontane beech forests under Mts. Jalovnik and Krikov Vrh, on gullied slopes on mixed geological bedrock dominated by chert, and these stands are classified into the association Calamagrostio arundinaceae-Sorbetum ariae. While occupying only small areas these two pioneer stages, as the sites of some rare or protected species, are nevertheless important biotopes and play a vital role in protection against avalanches.
\end{abstract}

\section{Izvleček}

V južnih Julijskih Alpah smo opisali dve združbi, v katerih sta v drevesni plasti dominantni vrsti iz rodu Sorbus in opozorili na dva sukcesijska niza v zaraščanju opuščenih kmetijskih površin (pašnikov, senožeti). V drugotni sukcesiji na nekdanjih subalpinskih pašnikih nad planino Razor in pod grebenom Breginjskega Stola, kjer je potencialno naravna vegetacija subalpinski bukov gozd, je rušje prerasla jerebika (Sorbus aucuparia) in njene sestoje uvrščamo v novo asociacijo Rhododendro hirsuti-Sorbetum aucupariae. Na strmih nekdanjih senožetih v pasu altimontanskih bukovih gozdov pod Jalovnikom in Krikovim vrhom pa se na mešani geološki podlagi, kjer prevladuje roženec, v užlebljenih pobočjih uveljavi mokovec (Sorbus aria) in njegove sestoje uvrščamo v asociacijo Calamagrostio arundinaceae-Sorbetum ariae. Opisana pionirska stadija imata kljub majhnim površinam pomembno varovalno vlogo pred snežnimi plazovi in biotopski pomen, kot rastišča nekaterih redkih ali zavarovanih vrst.

1 Institute of Biology, Scientific Research Centre of the Slovenian Academy of Sciences and Arts, Regional unit Tolmin, Brunov drevored 13, SI-5220 Tolmin, and Biotechnical Faculty of the University in Ljubljana, Department of Forestry and Renewable Forest Resources, Večna pot 83, SI-1000 Ljubljana.

E-mail: Igor.Dakskobler@zrc-sazu.si 


\section{Introduction}

Several years ago we described a pioneer community of mountain ash (Sorbus aucuparia) and green alder (Alnus viridis) - Alno viridis-Sorbetum aucupariae on former grazing areas and abandoned mountain pastures in the belt of altimontane and subalpine beech forests in the southern Julian Alps and their foothills (Dakskobler et al. 2013). In 2014 and 2015 we observed a similar forest type in these mountains, with dominating mountain ash in the tree layer and dominating dwarf pine (Pinus mugo) in the shrub layer. Such stands, also of pioneer origin, were found in the level ground $\mathrm{Na}$ Polju above the alp Planina Razor and under the ridge of Breginjski Stol. Presumably, there used to be beech forest that was later burned and turned into pastures. These were abandoned decades ago and subsequently dwarf pine was the first to establish itself in the succession, followed by mountain ash. Another form of pioneer forest on former agricultural areas was observed on sunny slopes of Mt. Jalovnik above the village Sela nad Podmelcem and un- der Mt. Krikov Vrh above the alp Kuk (Podkuk). Steep hay meadows here have been overgrown with mixed forest whose tree layer is dominated by whitebeam (Sorbus aria). Potential natural vegetation on these sites as well is altimontane beech forest. Given that both mountain ash and whitebeam only rarely form forest stands in the Julian Alps we decided to phytosociologically describe their communities.

\section{Methods}

Applying the Central-European phytosociological method (Braun-Blanquet 1964) we studied the pioneer forest stands of mountain ash and dwarf pine above the alp Planina Razor, under the Breginjski Stol ridge as well as grasslands and whitebeam stands under Mts. Jalovnik and Krikov Vrh (Figures 1, 2, 6, 7, 8, 9, 10 and 11). Relevés were entered into the FloVegSi database (Seliškar \& al. 2003). Combined cover-abundance values were transformed into numerical values 1-9 (van der Maarel 1979).

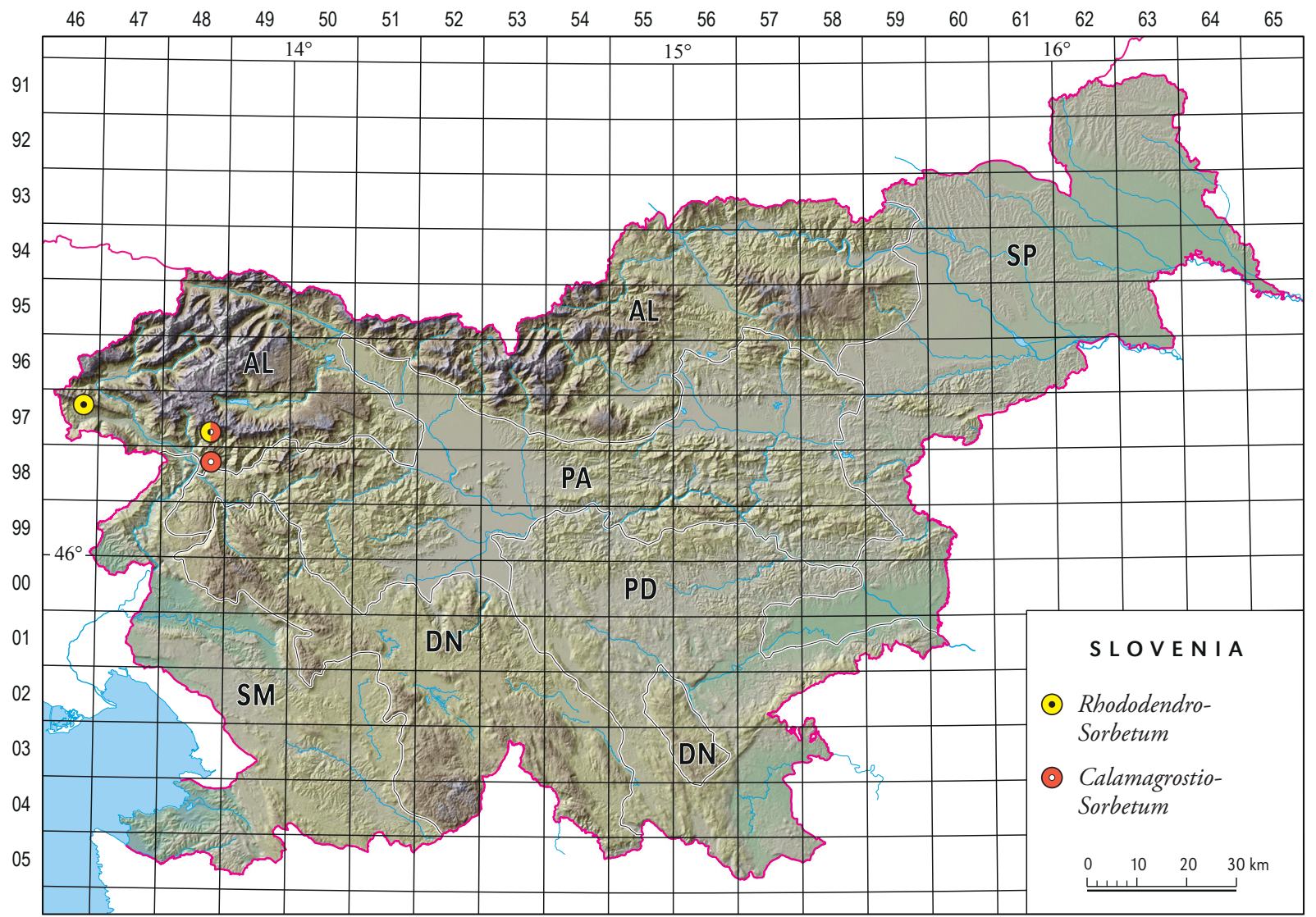

Figure 1: Research area on the map of Slovenia (AL - Alpine phytogeographical region, PA - pre-Alpine phytogeographical region, SM - subMediterranean phytogeograpahical region, DN - Dinaric phytogeographical region, PD - pre-Dinaric phytogeographical region, SP-sub-Pannonian phytogeographical region).

Slika 1: Raziskovano območje na zemljevidu Slovenije. 
Numerical comparisons were conducted with the software package SYN-TAX (Podani 2001). The relevés were arranged in three analytical tables by means of hierarchical classification. The nomenclature source for the names of vascular plants is the Mala flora Slovenije (Martinčič et al. 2007), Martinčič $(2003,2011)$ is the nomenclature source for the names of mosses, Suppan et al. (2000) for the names of lichens. The nomenclature sources for the names of syntaxa are Šilc \& Čarni (2012), Dakskobler et al. (2013) and Zupančič (2013).

\section{Results and Discussion}

\section{Sorbus aucuparia community above the alp Planina Razor and under the Breginjski Stol ridge}

Table 1 comprises nine relevés made under the ridge of the Breginjski Stol above the Učja valley and at the karstified plateau (level ground) Na Polju above the alp Planina Razor (Figure 2). One of the relevés under Stol

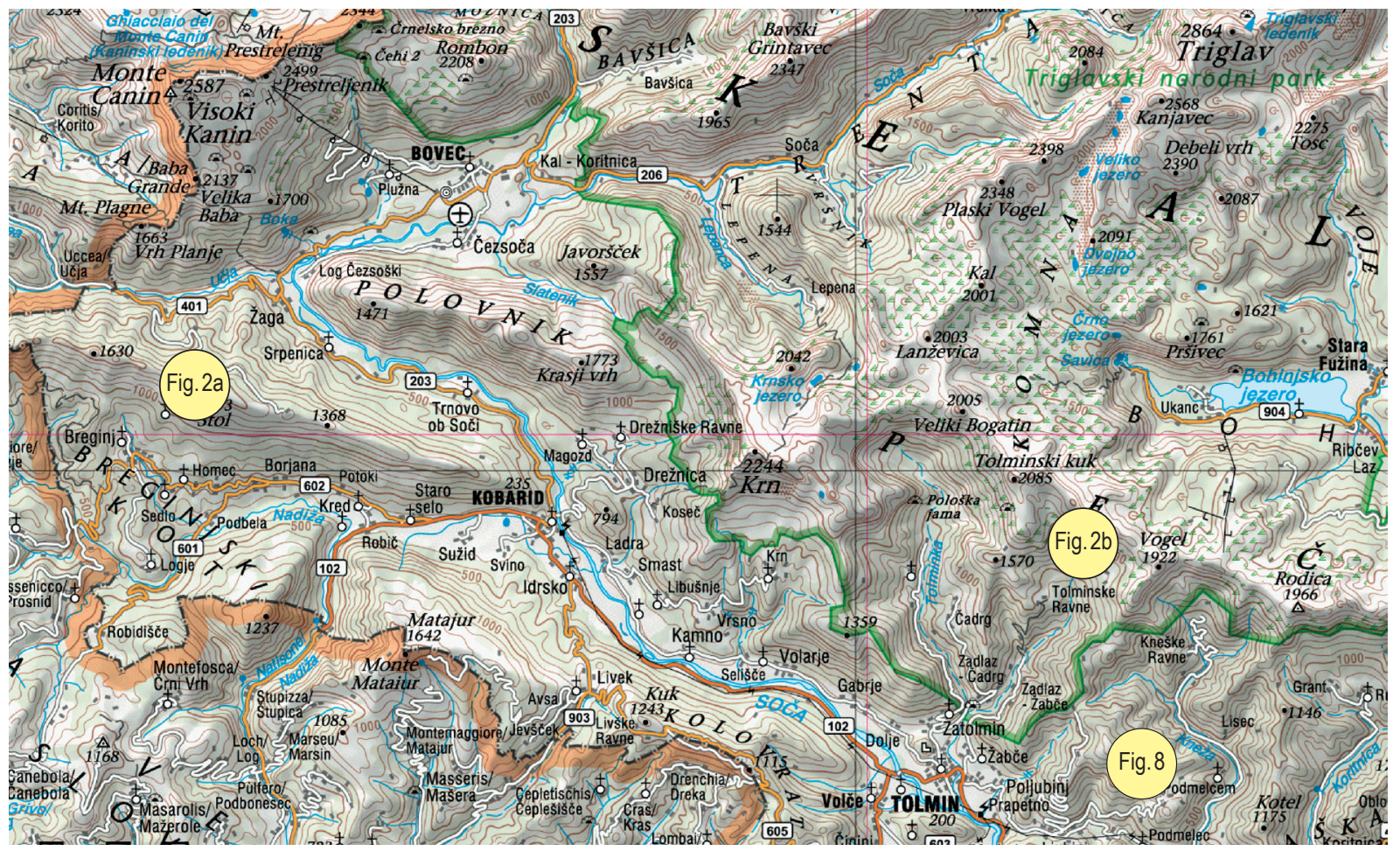

Figure 2: Localities of Sorbus aucuparia (figures 2a and 2b) and Sorbus aria (figure 8, p. 73) stands in the southern Julian Alps (western Slovenia) Slika 2: Nahajališ̌̌a popisanih sestojev jerebike (sliki 2a in 2b) in mokovca (slika 8 na strani 73) v južnih Julijskih Alpah (zahodna Slovenija).

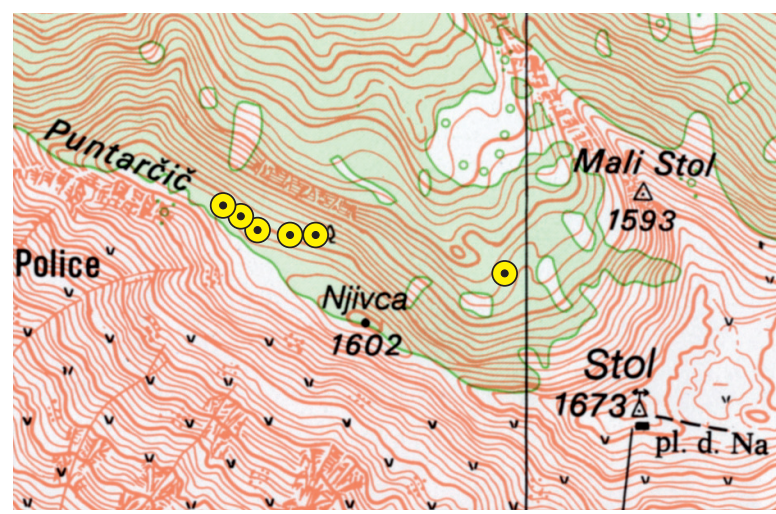

Figure 2a: Localities of Sorbus aucuparia stands under the Breginjski Stol ridge.

Slika 2a: Nahajališča popisanih sestojev jerebike pod grebenom

Breginjskega Stola.

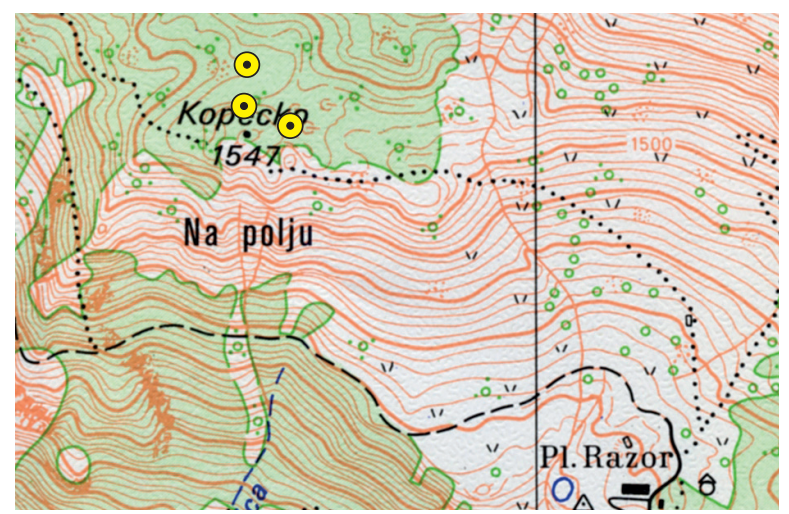

Figure 2b: Localities of Sorbus aucuparia stands above the alp Planina Razor.

Slika 2b: Nahajališča popisanih sestojev jerebike nad pl. Razor. 
was made above the cirque Dol at the elevation of around $1460 \mathrm{~m}$ and five under the ridge Puntračič-Njivca, on the location of a former pasture, at the elevation of $1540 \mathrm{~m}$ to $1550 \mathrm{~m}$. The geological bedrock is Triassic Dachstein limestone (Buser 1986, 1987). These are potentially the sites of subalpine beech forest (Polysticho lonchitis-Fagetum) that continues into dwarf pine stands (Rhodothamno-Pinetum mugo) on the ridge.

$\mathrm{Na}$ Polju is a rectangular level terrain at the elevation of about $1530 \mathrm{~m}$, measuring $700 \mathrm{~m} \times 300 \mathrm{~m}$ (Kunaver 1993: 38). We made three relevés here. The geological bedrock is Triassic Dachstein limestone and in the western part of the level also breccia (Buser 1986, 1987, Kunaver, ibid.). Previously occupied by pastures, this level ground is now mainly overgrown with dwarf pine stands. Especially prominent in the northwestern part of this level ground, which is also the most basin-shaped, is the Sorbus aucuparia forest, which can be seen from afar.

The floristic composition of these nine relevés was compared to ten relevés of similar Sorbus aucuparia stands that were recorded under Mt. Matajur and above the upper Bača Valley and classified into the association Alno viridis-Sorbetum aucupariae, namely into two subassociations: -luzuletosum sylvaticae on deep, slightly acid soil, and -adenostyletosum glabrae on moist stony sites (Dakskobler et al. 2013). We determined that these mountain ash stands are a stage in the secondary succession on potential sites of altimontane or subalpine beech forest. The successional sere proceeds in the following manner:

Beech forest (Ranunculo platanifolii-Fagetum, Polysticho lonchitis-Fagetum) - pasture (hay meadow) - green alder stands (Rhododendro hirsuti-Alnetum viridis, Alnetum viridis) - mountain ash stands (Alno viridis-Sorbetum aucupariae) - beech forest.

We compared cover values of species (Figure 3) and presence or absence of species separately (Figure 4). The results indicate that relevés from Table $1(\mathrm{RhSa})$ group separately from the relevés of the association Alno viridisSorbetum aucupariae (AvSa) and therefore cannot be classified into this association. Three relevés of the association Alno viridis-Sorbetum aucupariae that belong to the subassociation luzuletosum sylvaticae stand out somewhat in terms of floristic composition.

Similar conclusions can be made based on the synthetic table (Table 2), where we took into account the rank of the subassociation. The first two columns in this table show the composition of two subassociations of Alno viridis-Sorbetum aucupariae (AvSals = subass. luzuletosum sylvaticae;AvSaag = subass. adenostyletosum glabrae) and columns 3 and 4 comprise the relevés under the Breginjski Stol (RhSadc) and from the plateau $\mathrm{Na}$ Polju (RhSacv). Thess four syntaxa (columns) were

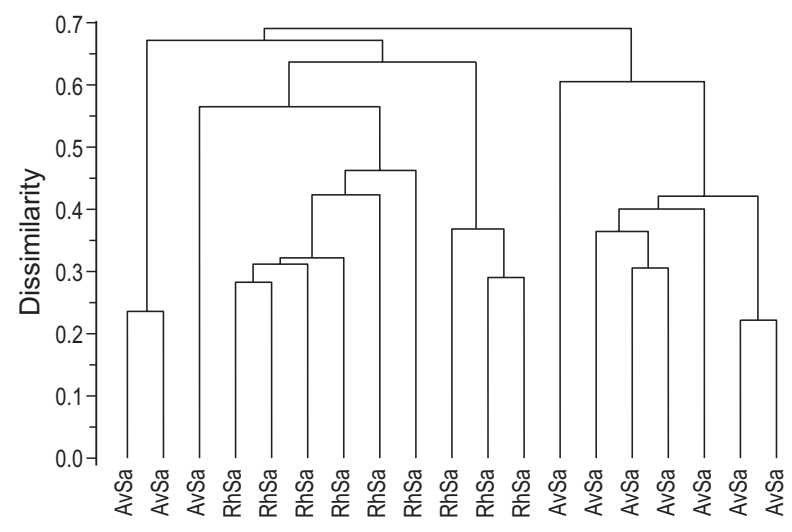

Figure 3: Dendrogram of Sorbus aucaparia communities in the southern Julian Alps (AvSa = Alno viridis-Sorbetum aucupariae; $\mathrm{RhSa}=$ Rhododendro hirsuti-Sorbetum aucupariae), UPGMA, similarity ratio.

Slika 3: Dendrogram jerebikovih združb v južnih Julijskih Alpah (AvSa = Alno viridis-Sorbetum aucupariae RhSa $=$ Rhododendro hirsuti-Sorbetum aucupariae), UPGMA, similarity ratio.

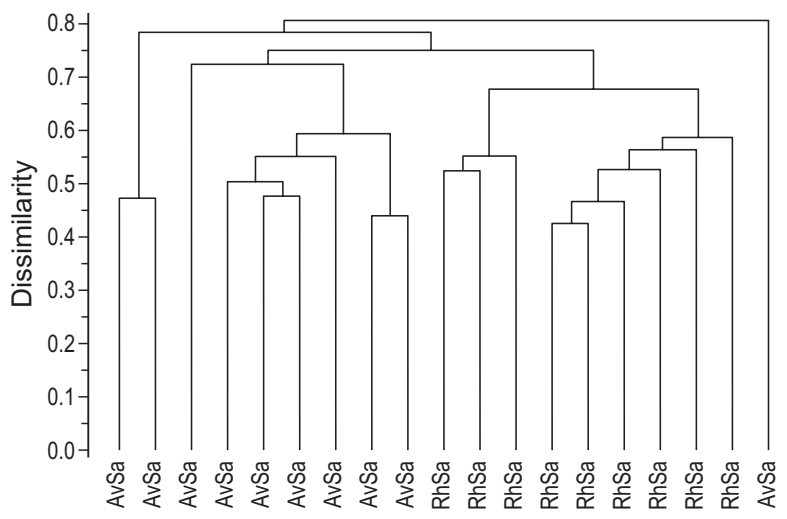

Figure 4: Dendrogram of Sorbus aucaparia communities in the southern Julian Alps $(\mathrm{AvSa}=$ Alno viridis-Sorbetum aucupariae; $\mathrm{RhSa}=$ Rhododendro hirsuti-Sorbetum aucupariae), UPGMA, Jaccard.

Slika 4: Dendrogram jerebikovih združb v južnih Julijskih Alpah (AvSa = Alno viridis-Sorbetum aucupariae RhSa $=$ Rhododendro hirsuti-Sorbetum aucupariae), UPGMA, Jaccard.

compared with hierarchical classification and the results are in Figure 5.

Despite certain similarities - the stands of the subassociation Alno-Sorbetum luzuletosum also comprise the shrub species Sorbus chamaemespilus and in places Pinus mugo, in some spots within the stands of the subassociation Alno-Sorbetum adenostyletosum we can observe also Rhododendron hirsutum and many species of stony montane forests - it is clear that the mountain ash stands under the Breginjski Stol ridge and above the alp Planina Razor cannot be classified into the association Alno viridis-Sorbetum, in the first place because green alder does not even occur within them. The composition by 


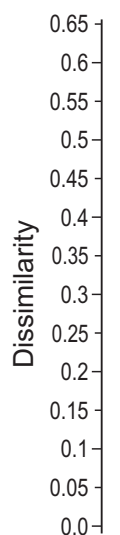

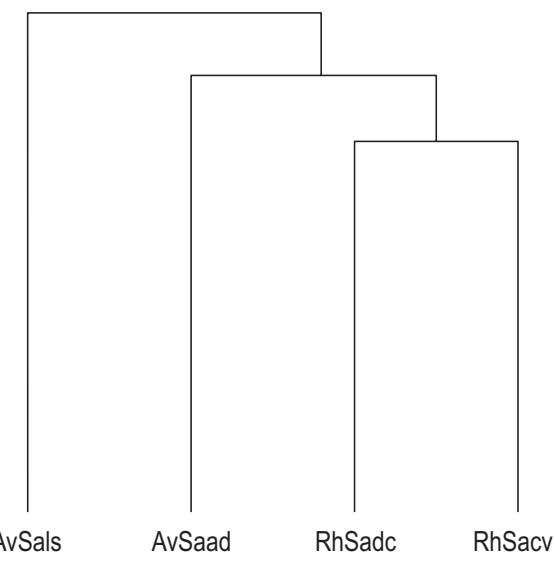

Figure 5: Dendrogram of Sorbus aucaparia communities in the southern Julian Alps (AvSals = Alno viridis-Sorbetum aucupariae luzuletosum sylvaticae; AvSaad = Alno viridis-Sorbetum aucupariae adenostyletosum glabrae; $\mathrm{RhSadc}=$ Rhododendro hirsuti-Sorbetum aucupariae deschampsietosum cespitosae, $\mathrm{RhSacv}=$ Rhododendro hirsuti-Sorbetum aucupariae calamagrostietosum variae), UPGMA, similarity ratio.

Slika 5: Dendrogram jerebikovih združb v južnih Julijskih Alpah (AvSals = Alno viridis-Sorbetum aucupariae luzuletosum sylvaticae; AvSaad = Alno viridis-Sorbetum aucupariae adenostyletosum glabrae; $\mathrm{RhSadc}=$ Rhododendro hirsuti-Sorbetum aucupariae deschampsietosum cespitosae; $\mathrm{RhSacv}=$ Rhododendro hirsuti-Sorbetum aucupariae calamagrostietosum variae), UPGMA, similarity ratio. groups of diagnostic species (Table 3) indicates differences within subassociations rather than differences at the level of associations. In terms of proportion the researched stands (columns 3 and 4 in Table 3 ) are clearly dominated by species of the classes Vaccinio-Piceetea and Erico-Pinetea, so they should be classified into the class Vaccinio-Piceetea or, if considering also their physiognomy (the structure of stands), also to the class Roso pendulinae-Pinetea mugo.

The successional sere is different as well and can be described as follows:

Subalpine beech forest (Polysticho lonchitis-Fagetum) - pasture, grassland (Ranunculo hybridi-Caricetum sempervirentis) - dwarf pine stands (Rhodothamno-Pinetum mugo) - mountain ash stands (Rhododendro hirsuti-Sorbetum aucupariae) - subalpine beech stands.

This successional sere indicates the sequence in secondary succession when a former subalpine pasture becomes overgrown through dwarf pine into a forest whose final stage will be beech. This mountain ash community is therefore classified into a new association Rhododendro hirsuti-Sorbetum aucupariae ass. nov. hoc loco. Its nomenclature type, holotypus, is relevé No. 1 in Table 1. Diagnostic species of the new association are Sorbus au-

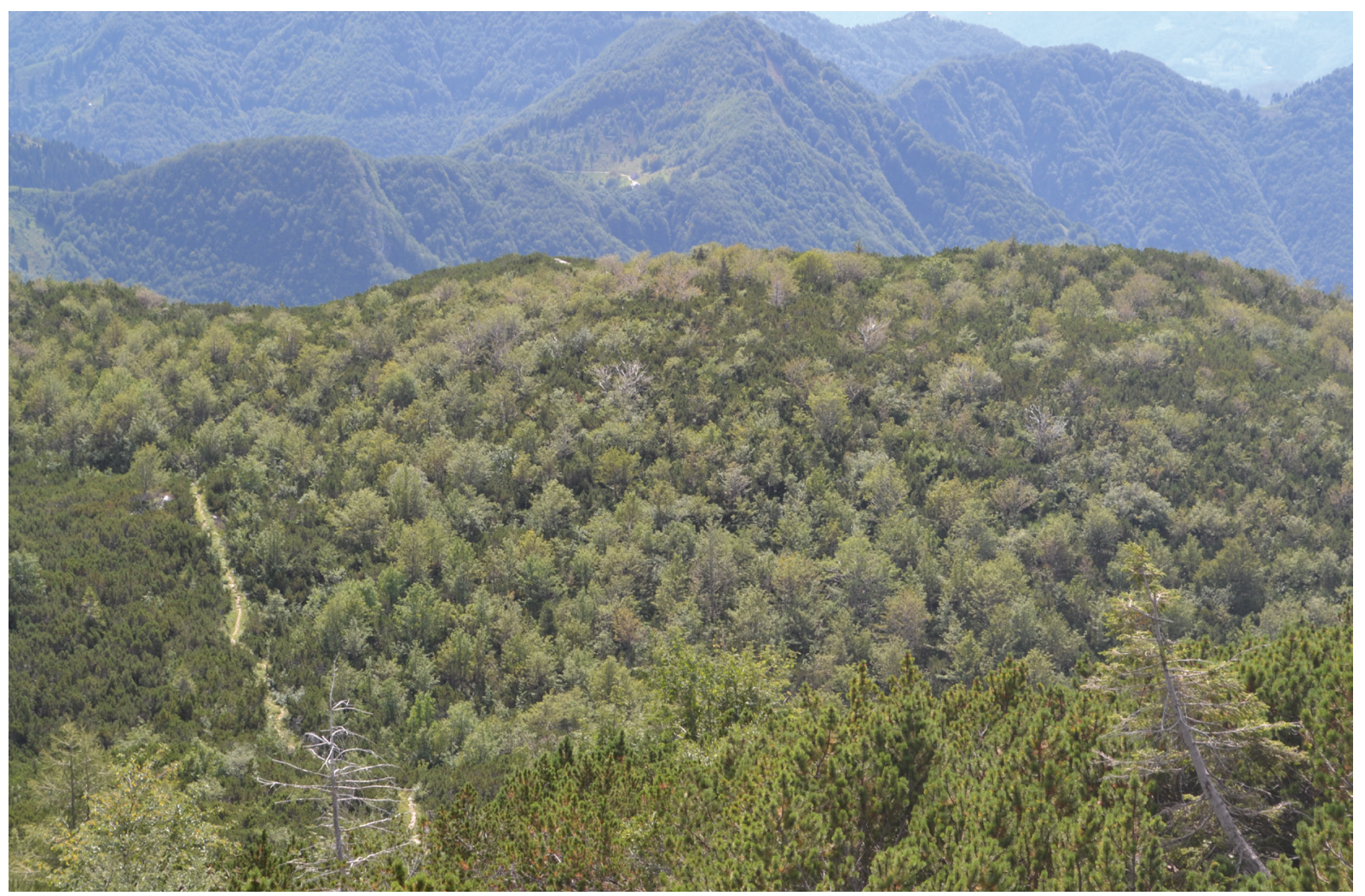

Figure 6: Level ground Na Polju with Pinus mugo and Sorbus aucuparia stands.

Slika 6: Uravnava Na Polju s ruševjem in jerebikovjem. 
cuparia subsp. aucuparia, Lycopodium annotinum, Pinus mugo, Salix waldsteiniana and Rhododendron hirsutum. The listed species well differentiate the studied community against the physiognomically similar community Alno viridis-Sorbetum aucupariae and indicate its close syndynamic relationship with dwarf pine stands from the association Rhodothamno-Pinetum mugo. However, it cannot be classified within this association as we should consider the dominant species of the highest stand layer - the tree layer, which is obviously mountain ash. The syntaxonomical rank of the subassociation Rhodothamno-Pinetum mugo sorbetosum aucupariae is therefore inappropriate in the case of mountain ash forest. For now, the new association is classified into the alliance Erico-Pinion mugo, order Junipero-Pinetalia and class Vaccinio-Piceetea. In terms of physiognomy it could also be classified into the alliance Erico-Pinion mugo, order Junipero-Pinetalia and class Roso pendulinae-Pinetea mugo.

Stands under the Breginjski Stol ridge are classified into the new subassociation Rhododednro hirusti-Sorbetum aucupariae deschampsietusum cespitosae. Its differential species are Deschampsia cespitosa, Lonicera nigra, Myosotis sylvatica agg. Molopospermum peloponesiacum subsp. bauhinii, Primula elatior and Calamagrostis arundinacea. They indicate fresher sites with deeper and slightly acidic soils, as well as a certain similarity with the stands of the association Alno viridis-Sorbetum aucupariae. Its nomenclature type, holotypus, is relevé No. 1 in Table 1.

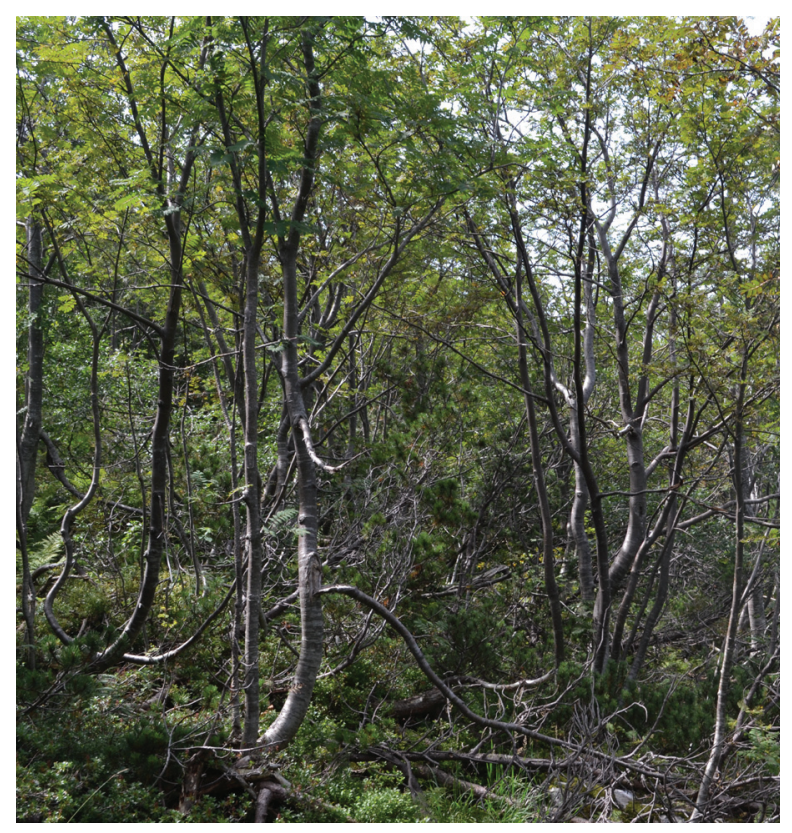

Stands above the alp Planina Razor are classified into the new subassociation Rhododendro hirusti-Sorbetum aucupariae calamagrostietosum variae. Its differential species are Calamagrostis varia, Rhodothamnus chamaecistus and Horminum pyrenaicum. They indicate stony calcareous sites in cold Alpine climate, and Horminum pyrenaicum also a connection with former pastures. Its nomenclature type, holotypus, is relevé No. 8 in Table 1.

\section{Sorbus aria community on abandoned hay-fields under Mts. Jalovnik and Krikov Vrh}

Krikov Vrh is a $1298 \mathrm{~m}$-high mountain in the lateral ridge of the southern Julian Alps in direction from Žabijski Kuk towards Tolminski Triglav. The parent material is mixed, dominated by platy limestone with marl and chert, and Bača dolomite with chert (Buser 1986, 1987). Its shady and partly also sunny slopes are overgrown with montane-altimontane forests from the associations Homogyno sylvestris-Fagetum, Saxifrago cuneifolii-Fagetum, Fraxinio orni-Ostryetum and Amelanchiero ovalis-Pinetum mugo (Dakskobler 2002, 2014, 2015). There are some abandoned hay meadow on the shady side under the peak, and to the north of the peak there is alp Kuk (Podkuk), which is still being used as a pasture. The phytosociological composition of the pioneer Sorbus aria stand on an abandoned hay meadow under Krikov $\mathrm{Vrh}$ is shown in relevé 5 in Table 5.

Jalovnik is a $1452 \mathrm{~m}$-high mountain in the foothills of the southern Julian Alps. The shady slopes above the Kneža valley are forested with predominantly beech forests from the associations Ranunculo platanifolii-Fagetum and Homogyno sylvestris-Fagetum, only the former pastures under the peak of the mountain are now becoming overgrown with green alder (Alnus viridis). Except for the rockiest parts (dominated by the stands of the associations Ostryo-Fagetum and Fraxino orni-Ostryetum), the sunny slopes have been cleared (or burned) for pastures and hay meadows. Most of them have been abandoned for a long time. The species composition of these former hay meadows is presented in Table 4. Because of the mixed geological bedrock (limestone mixed with marlstone and chert, with the latter completely dominating some areas - Buser 1986, 1987) and slightly acid soil the character species of dry grasslands (class Festuco-Brometea), thermophilous forest edges (class Trifolio-Geranietea) and subalpine grasslands on calcareous bedrock (class

Figure 7: Stand of the subassociation Rhododendro hirsuti-Sorbetum aucupariae calamagrostietosum variae. 


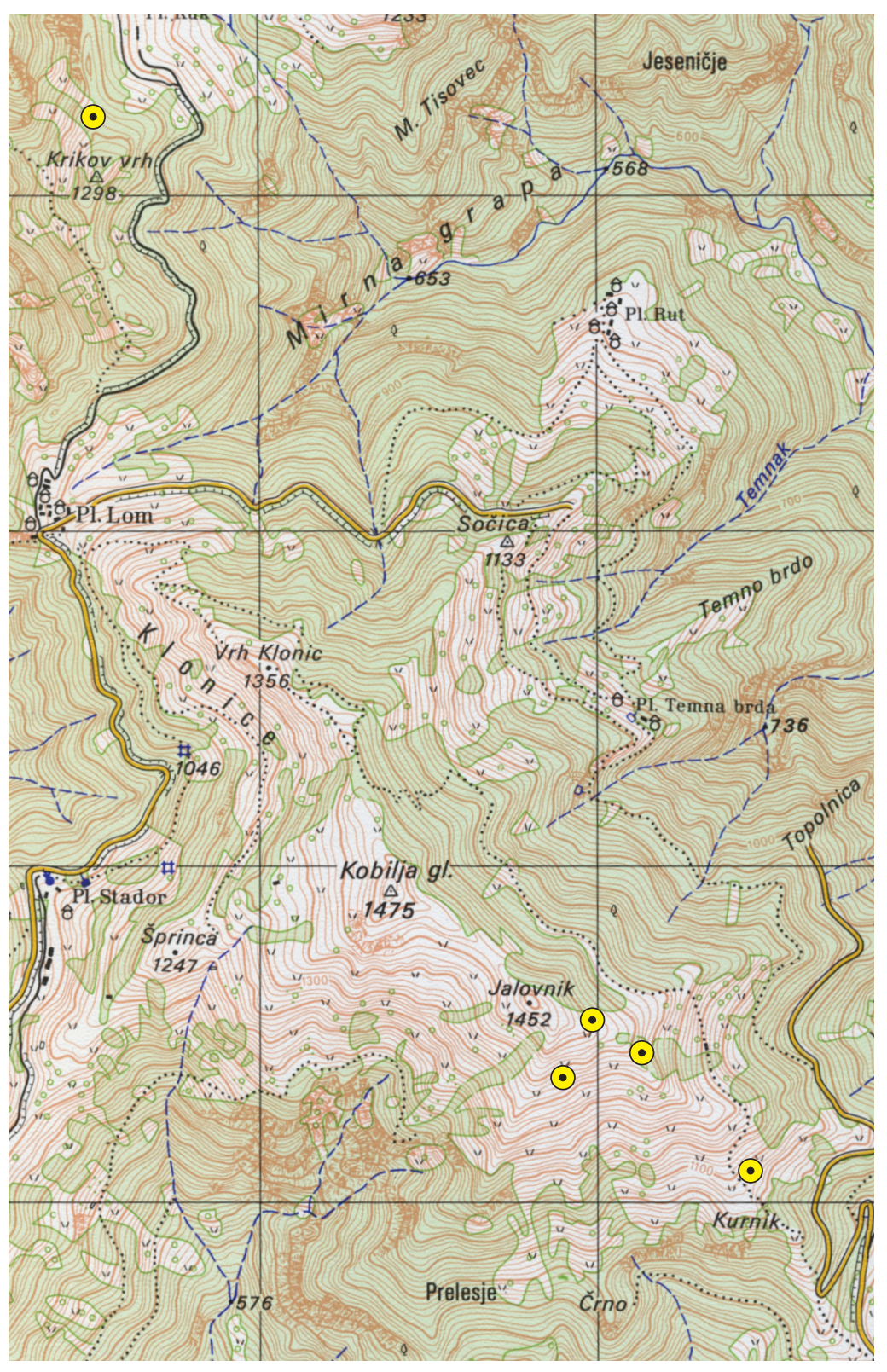

Figure 8: Localities of Sorbus aria stands under Mts. Jalovnik and Krikov Vrh.

Slika 8: Nahajališča sestojev mokovca pod Jalovnikom in Krikovim vrhom. and Centaureo julici-Geranietum sanguinei nom. prov. (relevés 4 to 7 in Table 4). The presence of shrub and tree species (Rosa canina. R. glauca, R.villosa, Sorbus aria, Berberis vulgaris, Juniperus communis, Rubus idaeus, Sorbus aria and S. chamaemespilus) indicates delayed overgrowing and gradual re-establishment of the forest. In some, especially gullied parts with predominating chert and deep, slightly acid soil, former hay meadows have already become overgrown with forest (its phytosociological structure is presented in Table 5, relevés $1-4$, relevé 5 represents the stand from Mt. Krikov Vrh). The tree layer of these pioneer stands is dominated by Sorbus aria, individual specimens of Sorbus aucuparia, Picea abies, Acer pseudoplatanus, Salix caprea, Betula pendula and, very rarely, also Larix decidua, Tilia platyphyllos and Fagus sylvatica. Rubus idaeus is very frequent in the shrub layer, while Calamagrostis arundinacea, Veratrum album subsp. lobelianum, Athyrium filix femina, Luzula luzuloides and Senecio ovatus frequently occur in the herb layer. These stands were recorded at the elevation between $1150 \mathrm{~m}$ to $1400 \mathrm{~m}$, on potential sites of the altimontane beech forest from the association Ranunculo platanifolii-Fagetum and partly from the association Saxifrago cuneifolii-Fagetum (Dakskobler 2015). The successional stage with the dominating Sorbus aria in the tree layer under Mts. Jalovnik and Krikov Vrh is classified into the new association Calamagrostio arundinaceae-Sorbetum ariae ass. nov. hoc loco. Diagnostic species of the new association are Sorbus aria, Calamagrostis arundinacea, Veratrum album subsp. lobelianum, Luzula luzuloides and Convallaria majalis. They indicate a special site ecology - frequently steep, sunny and shady gullied slopes in the altimontane belt on mixed geological bedrock with dominating chert and shallow, slightly acid and fresh (dystric or eutric) brown soil, with sites gradually changing back into the beech forest. Sorbus aria is a character species of the order Quercetalia pubescenti-petraeae and in Slovenia it occurs mainly in hop 


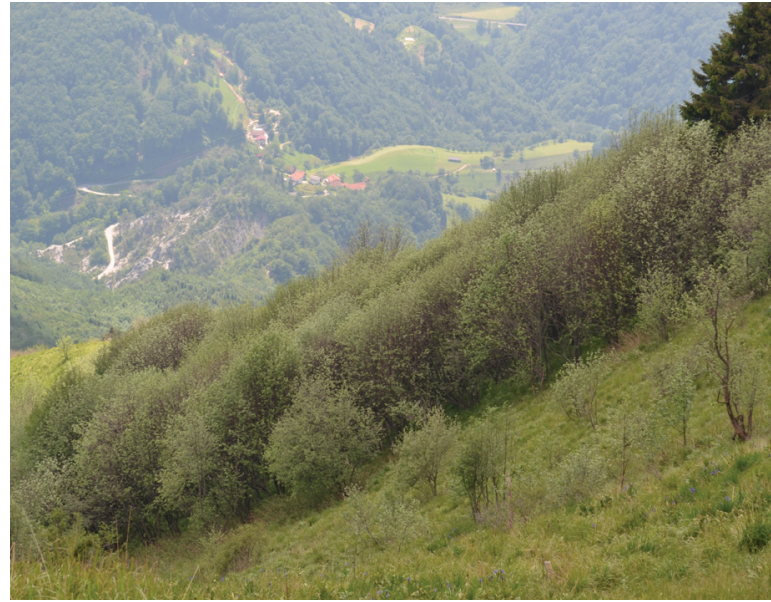

Figure 9: Sorbus aria stand on the sunny slopes of Mt. Jalovnik. Slika 9: Sestoj mokovca na prisojnih pobočjih Jalovnika.

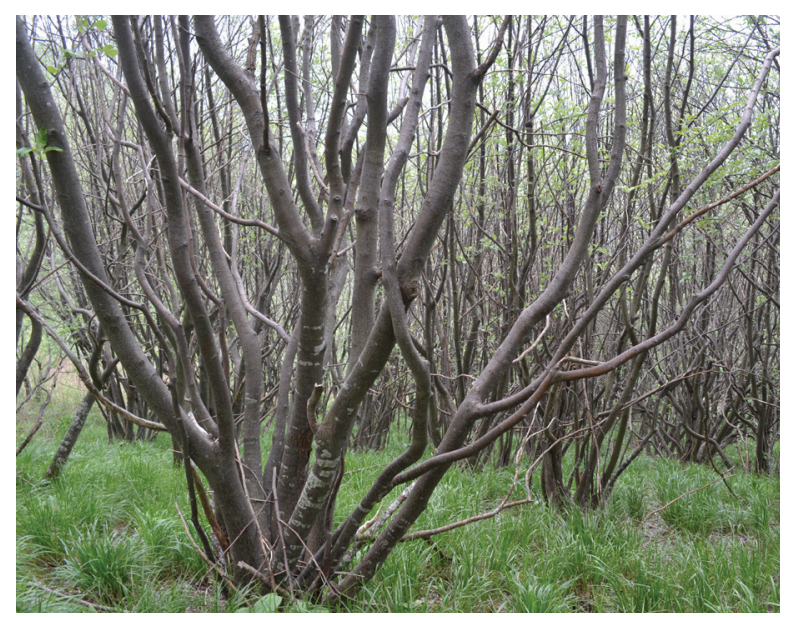

Figure 10: Stand of the association Calamagrostio arundinaceaeSorbetum ariae.

Slika 10: Sestoj asociacije Calamagrostio arundinaceae-Sorbetum ariae.

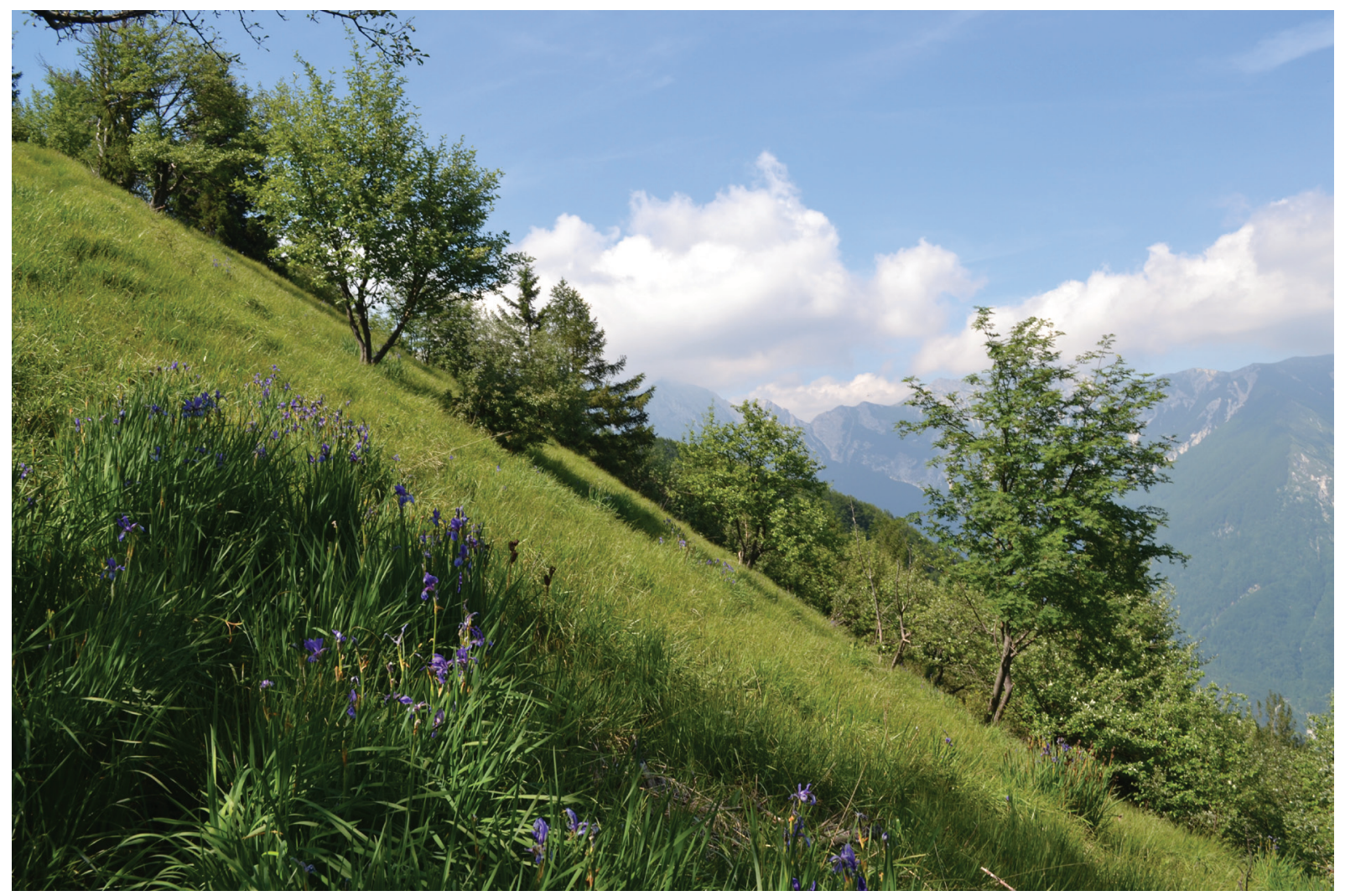

Figure 11: Former hay-fields on the sunny slopes of Mt. Jalovnik with Iris sibirica subsp. erirrhiza.

Slika 11: Nekdanje senožeti pod Jalovnikom s taksonom Iris sibirica subsp. erirrhiza.

hornbeam, beech and pine forests. The nomenclature type of the new association, holotypus, is relevé No. 2 in Table 5. The new association is classified into the alliance Sambuco-Salicion capreae, order Sambucetalia and class Rhamno-Prunetea. The studied Sorbus aria stands under
Mts. Jalovnik and Krikov Vrh can still be characterised as a pioneer forest on fresh sites in the montane belt, where the tree layer is dominated by Salix caprea, Populus tremula, Betula pendula and (or) Sorbus aucuparia (Exner \& Willner 2007). 


\section{Conclusions}

Stands with the tree layer dominated by Sorbus aucuparia and (or) Sorbus aria are rare in Slovenia. These two species usually occur in the lower tree layer of oak, hop-hornbeam, pine, larch, beech and (or) spruce communities. Both heliophilous pioneers can, in special site conditions in certain successional stages, dominate in the upper stand layer. We conducted a phytosociological study into two such examples from the southern Julian Alps. In the first, on former subalpine pastures on the level $\mathrm{Na}$ Polju above the alp Planina Razor and under the Breginjski Stol ridge, mountain ash (Sorbus aucuparia) has overgrown dwarf pine (Pinus mugo). In the second, on former hay meadows on chert under Mt. Jalovnik above the village Sela nad Podmelcem and under Mt. Krikov Vrh, turf species such as Calamagrostis arundinacea and Molinia caerulea subsp. arundcinacea have been overgrown by Sorbus aria. Even though such pioneer forests tend to be relatively quickly (but not earlier than in half a century) replaced by climax species, in our case beech, they should not be overlooked, especially given their protective function (protection against avalanches) as well as their role as the sites of some endemic, rare or protected species (Anon. 2002, 2004). Stands of the association Rhododendro hirsuti-Sorbetum aucupariae belong to the habitat type of Community interest $4070^{*}$ Bushes with Pinus mugo and Rhododendretum hirsutum and comprise also protected or rare species such as Lycopodium annotinum, Huperzia selago, Horminum pyrenaicum, Athyrium distentifolium, Gentiana pannonica, Cyclamen purpurascens, Lilium martagon, and Aconitum angustifolium. Iris sibirica subsp. erirrhiza is an example of such species in the stands of the association Calamagrostio arundinaceae-Sorbetum ariae. This southeastern-Alpine-Illyrian taxon is more frequent on former, contact hay meadows that comprise also protected or rare species and endemics like Arnica montana, Gymnadenia conopsea, Dianthus hyssopifolius, Traunsteinera globosa, Lilium martagon, L. carniolicum, Aconitum angustifolium, Platanthera bifolia, Veratrum nigrum, Rosa villosa, Helleborus odorus and Centaurea haynaldii subsp. julica.

Synsystematic classification of the studied communities into higher units is as follows:

Vaccinio-Piceetea Br.-Bl. et al. 1939 em. Zupančič (1976) 1980

Junipero-Pinetalia Boşcaiu 1971

Erico-Pinion mugo Leibundgut 1948

Rhodothamno-Pinenion mugo Zupančič 2013

Rhododendro hirsuti-Sorbetum aucupariae ass. nov.
Possible is also classification according to Šilc \& Čarni (2012):

Roso pendulinae-Pinetea mugo Theurillat in Theurillat et al. 1995

Junipero-Pinetalia Boşcaiu 1971

Erico-Pinion mugo Leibundgut 1948

Rhododendro hirsuti-Sorbetum aucupariae ass. nov.

Calluno-Ulicetea Br.-Bl. et Tx. ex Klika 1948

Nardetalia strictae Preising 1950

Nardo-Agrostion tenuis Sillinger 1933

Homogyno alpinae-Nardetum Mráz 1956

Festuco-Brometea Br.-Bl. \& Tx. ex Soó 1947

Brometalia erecti Koch 1926

Bromion erecti Koch 1926

Centaureo julici-Geranietum sanguinei nom. prov.

Molinio arundinaceae-Iridetum erirrhizae nom. prov.

Rhamno-Prunetea Rivas Goday et Borja Carbonell ex Tx. 1962

Sambucetalia racemosae Oberd. ex Doing 1962

Sambuco-Salicion capreae Tx. et Neumann ex Oberd. 1957

Calamagrostio arundinaceae-Sorbetum ariae ass. nov.

\section{Povzetek}

\section{Dve novi pionirski združbi drevesnih vrst Sorbus au- cuparia in Sorbus aria v južnih Julijskih Alpah}

Sestoji, v katerih v drevesni plasti prevladujeta vrsti Sorbus aucuparia in (ali) Sorbus aria, so v Sloveniji precejšnja redkost. Navadno sta ti dve vrsti primešani v spodnji drevesni plasti hrastovih, črnogabrovih, borovih, macesnovih, bukovih in (ali) smrekovih združb. Oba svetloljubna pionirja $\mathrm{v}$ posebnih rastiščnih razmerah $\mathrm{v}$ nekaterih sukcesijskih stadijih lahko prevladata $\mathrm{v}$ zgornji sestojni plasti. Fitocenološko smo preučili dva taka primera iz južnih Julijskih Alp. V prvem, na nekdanjih visokogorskih pašnikih na potencialnih rastiščih subalpinskega bukovja (Polysticho lonchitis-Fagetum) na uravnavi Na polju nad pl. Razor in pod grebenom Breginjskega Stola, je jerebika (Sorbus aucuparia) prerasla rušje (Pinus mugo) in njene sestoje uvrščamo v novo asociacijo Rhododendro hirsuti-Sorbetum aucupariae. V drugem primeru, na nekdanjih senožetih na rožencu pod Jalovnikom nad Selmi nad Podmelcem in pod Krikovim vrhom pri planini Kuk (na potencialnih rastiščih altimiontanskega bukovja) pa je travno rušo vrst kot sta Calamagrostis arundinacea in Molinia caerulea 
subsp. arundcinacea zarasel mokovec in njegova sestoje uvrščamo v novo asociacijo Calamagrostio arundinaceae-Sorbetum ariae. Kljub temu, da takšne pionirske gozdove navadno razmeroma hitro (a ne prej kot $\mathrm{v}$ pol stoletja) nadomesti klimaksna vrsta, v našem primeru bukev, je nanje smiselno opozoriti, predvsem zaradi varovalne vloge, ki jo opravljajo (zaščita pred snežnimi plazovi), a tudi kot rastišča nekaterih endemičnih, redkih ali zavarovanih vrst (Anon. 2002, 2004). Sestoji asociacije Rhododendro hirsuti-Sorbetum aucupariae sodijo v evropsko varstveno pomemben habitatni tip $4070^{*}$ Ruševje z dlakavim slečem (Mugo-Rhododendretum hirsuti) in v njih uspevajo tudi zavarovane ali redke vrste, kot so Lycopodium annotinum, Huperzia selago, Horminum pyrenaicum, Athyrium distentifolium, Gentiana pannonica, Lilium martagon, $C y-$ clamen purpurascens in Aconitum angustifolium. V sestojih asociacije Calamagrostio arundinaceae-Sorbetum ariae je taka vrsta Iris sibirica subsp. erirrhiza. Ta jugovzhodnoalpsko-ilirski takson je sicer bolj pogost na stičnih nekdanjih senožetih, na katerih rastejo tudi zavarovane ali redke vrste ter endemiti Arnica montana, Gymnadenia conopsea, Dianthus hyssopifolius, Traunsteinera globosa, Lilium martagon, L. carniolicum, Aconitum angustifolium, Platanthera bifolia, Veratrum nigrum, Rosa villosa, Helleborus odorus in Centaurea haynaldii subsp. julica.

\section{Acknowledgements}

Iztok Sajko prepared Figures 1, 2 and 8 for print. Two anonymous reviewers helped us with valuable improvements and corrections. English translation by Andreja Šalamon Verbič.

\section{References}

Anonymus 2002: Pravilnik o uvrstitvi ogroženih rastlinskih in živalskih vrst v rdeči seznam. Priloga 1: Rdeči seznam praprotnic in semenk (Pteridophyta \& Spermatophyta). Uradni list RS 82/2002, pp. 8893-8910.

Anonymous 2004: Uredba o zavarovanih prosto živečih rastlinskih vrstah. Uradni list RS 46/2004.

Braun-Blanquet, J. 1964: Pflanzensoziologie. Grundzüge der Vegetationskunde. 3. Auflage. Springer, Wien - New York, 865 pp.

Buser, S. 1986: Tolmač listov Tolmin in Videm (Udine). Osnovna geološka karta SFRJ 1 : 100 000. Zvezni geološki zavod, Beograd, 103 pp.

Buser, S. 1987: Osnovna geološka karta SFRJ. Tolmin in Videm $1: 100$ 000. Zvezni geološki zavod, Beograd.

Dakskobler, I. 2002: Jelovo-bukovi gozdovi v dolinah Kneže, Zadlaščice in Tolminke (južne Julijske Alpe, zahodna Slovenija). Razprave 4. razreda SAZU 43-3: 111-165.
Dakskobler, I. 2014: Association Amelanchiero ovalis-Pinetum mugo in northwestern Slovenia. Acta Biologica Slovenica 57 (1): 15-43.

Dakskobler, I. 2015: Phytosociological analysis of montane beech forests on steep shady slopes on mixed geological bedrock in western Slovenia. Folia biologica et geologica 56 (1): 8-103.

Dakskobler, I., Rozman, A., Seliškar, A. 2013: Forest and scrub communities with green alder (Alnus viridis) in Slovenia. Hacquetia 12 (2): $95-185$.

Exner, A. \& Willner, W. 2007: Rhamno-Prunetea Rivas Goday \& Borja Carbonell ex Tx 1962. In: Willner, W. \& Grabherr, G. (eds.) 2007: Die Wälder und Gebüsche Österreichs. Ein Bestimmungswerk mit Tabellen. 1. Textband. Spektrum Akademischer Verlag in Elsevier, Heidelberg, pp. 62-83.

Kunaver, J. 1993: Prispevek k poznavanju pokrajine in geomorfologije dolin Tolminke in Zadlašce. In: Lipušček, R. (ur.): Dolini Tolminke in Zadlašce, ZOTK Slovenije in Triglavski narodni park, Tolmin, pp. 7-43

Maarel van der, E. 1979: Transformation of cover-abundance values in phytosociology and its effects on community similarity. Vegetatio 39 (2): $97-114$

Martinčič, A. 2003: Seznam listnatih mahov (Bryopsida) Slovenije. Hacquetia 2 (1): 91-166.

Martinčič, A. 2011: Seznam jetrenjakov (Marchanthiophyta) in rogovnjakov (Anthocerotophyta) Slovenije. Scopolia 72: 1-38.

Martinčič, A., Wraber, T., Jogan, N., Podobnik, A., Turk, B., Vreš, B., Ravnik, V., Frajman, B., Strgulc Krajšek, S., Trčak, B., Bačič, T., Fischer, M. A., Eler, K. \& Surina, B. 2007: Mala flora Slovenije. Ključ za določanje praprotnic in semenk. Četrta, dopolnjena in spremenjena izdaja. Tehniška založba Slovenije, Ljubljana, 967 pp.

Podani, J. 2001: SYN-TAX 2000. Computer Programs for Data Analysis in Ecology and Systematics. User's Manual, Budapest, 53 pp.

Seliškar, T., Vreš, B. \& Seliškar, A. 2003: FloVegSi 2.0. Fauna, Flora, Vegetation and Paleovegetation of Slovenia. Computer programme for arranging and analysis of biological data. Biološki inštitut ZRC SAZU, Ljubljana.

Suppan, U., Prügger, J. \& Mayrhofer, H. 2000: Catalogue of the lichenized and lichenicolous fungi of Slovenia. Bibliotheca Lichenologica 76: 1-215.

Šilc, U. \& Čarni, A. 2012: Conspectus of vegetation syntaxa in Slovenia. Hacquetia 11 (1): 113-164.

Zupančič, M., 2013: New considerations on southeast-Alpine and Dinaric-central Balkan dwarf pine. Hrvatska misao, 17 (1) / 13 (61) nova serija sv. 46, 156-172. 
Table 1: Rhododendro hirsuti-Sorbetum aucupariae.

Tabela 1: Rhododendro hirsuti-Sorbetum aucupariae.

\section{Number of relevé (Zaporedna številka popisa)}

Database number of relevé (Delovna številka popisa)

Elevation in $\mathrm{m}$ (Nadmorska višina $\mathrm{v} \mathrm{m})$

Aspect (Lega)

Slope in degrees (Nagib v stopinjah)

Parent material (Matična podlaga)

Soil (Tla)

Stoniness in \% (Kamnitost v \%)

Cover of tree layer in \% (Zastiranje drevesne plasti v \%)

Cover of upper shrub layer in \% (Zastiranje zgornje grmovne plasti v \%): E2b

Cover of lower shrub layer in \% (Zastiranje spodnje grmovne plasti v \%): E2a

Cover of herb layer in \% (Zastiranje zeliščne plasti v \%):

Cover of moss layer in \% (Zastiranje mahovne plasti v \%)

Maximum diameter of trees (Največji prsni premer dreves)

Maximum height of tress (Največja drevesna višina)

Number of species (Število vrst)

Relevé area (Velikost popisne ploskve)

Date of taking relevé (Datum popisa)

Quadrant (Kvadrant)

Coordinate GK X (D-48)

Coordinate GK X (D-48)

\section{$\begin{array}{lllllllll}1 & 2 & 3 & 4 & 5 & 6 & 7 & 8 & 9\end{array}$}

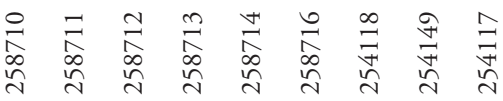

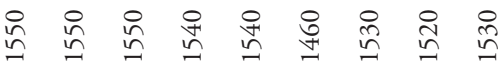

NE NE NE NNE NE NW NE SW N

$\begin{array}{lllllllll}20 & 20 & 25 & 25 & 20 & 30 & 10 & 10 & 15\end{array}$

A A A A A A A A A

$\operatorname{Re} \operatorname{Re} \operatorname{Re} \operatorname{Re} \operatorname{Re} \operatorname{Re} \operatorname{Re} \operatorname{Re} \operatorname{Re}$

$\begin{array}{lllllllll}5 & 5 & 0 & 5 & 5 & 20 & 20 & 20 & 20\end{array}$

$\begin{array}{llllllllll}\text { E3 } & 70 & 80 & 70 & 80 & 70 & 70 & 60 & 60 & 70\end{array}$

$\begin{array}{lllllllll}30 & 40 & 30 & 15 & 15 & 10 & 70 & 80 & 60\end{array}$

$\begin{array}{lllllllll}40 & 30 & 40 & 40 & 50 & 20 & 30 & 40 & 30\end{array}$

$\begin{array}{llllllllll}\text { E1 } & 80 & 80 & 80 & 80 & 80 & 80 & 60 & 80 & 70\end{array}$

$\begin{array}{llllllllll}\mathrm{E} 0 & 5 & 5 & 5 & 5 & 5 & 10 & 10 & 10 & 10\end{array}$

$\begin{array}{llllllllll}\mathrm{cm} & 25 & 20 & 20 & 35 & 30 & 25 & 20 & 25 & 30\end{array}$

$\begin{array}{llllllllll}\mathrm{m} & 10 & 10 & 8 & 10 & 10 & 10 & 8 & 10 & 14\end{array}$

$\begin{array}{lllllllll}56 & 53 & 48 & 57 & 48 & 75 & 46 & 64 & 78\end{array}$

$m^{2} \quad 400 \quad 400 \quad 400 \quad 400 \quad 400 \quad 400 \quad 400 \quad 400 \quad 400$

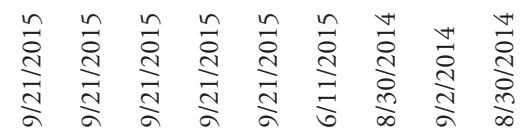

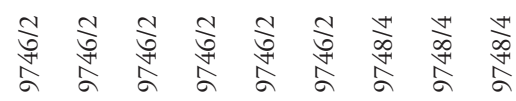

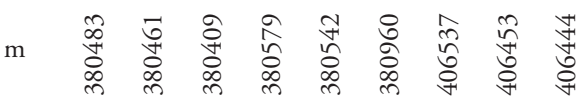

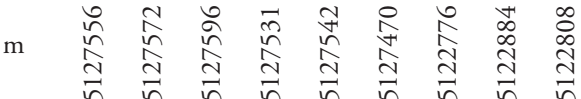

\begin{tabular}{|c|c|c|c|c|c|c|c|c|c|c|c|c|c|}
\hline & Diagnostic species of the association (Diagnostične vrste asociacije) & & & & & & & & & & & Pr. & Fr. \\
\hline SSC & Sorbus aucuparia & E3 & 4 & 4 & 4 & 5 & 4 & 4 & 4 & 4 & 4 & 9 & 100 \\
\hline SSC & Sorbus aucuparia & $\mathrm{E} 2 \mathrm{~b}$ & 1 & + & 1 & . & . & 1 & 1 & 1 & 1 & 7 & 78 \\
\hline SSC & Sorbus aucuparia & $\mathrm{E} 2 \mathrm{a}$ & . & + & + & . & + & 1 & . & + & + & 6 & 67 \\
\hline SSC & Sorbus aucuparia & E1 & . & . & . & + & . & . & . & + & + & 3 & 33 \\
\hline EP & Pinus mugo & $\mathrm{E} 2 \mathrm{~b}$ & 2 & 3 & 2 & 1 & 1 & 3 & 4 & 4 & 3 & 9 & 100 \\
\hline VP & Lycopodium annotinum & E1 & + & 1 & + & + & + & + & 1 & + & 3 & 3 & 33 \\
\hline EP & Rhododendron hirsutum & $\mathrm{E} 2 \mathrm{a}$ & + & . & . & + & + & 1 & 3 & 4 & 4 & 7 & 78 \\
\hline \multirow[t]{2}{*}{ BA } & Salix waldsteiniana & $\mathrm{E} 2 \mathrm{a}$ & + & + & . & 1 & 2 & + & + & $\mathrm{r}$ & . & 7 & 78 \\
\hline & Geographical differential species (Geografske razlikovalne vrste) & & & & & & & & & & & & \\
\hline $\mathrm{AF}$ & Anemone trifolia & E1 & + & + & . & + & . & + & . & . & . & 4 & 44 \\
\hline \multirow[t]{2}{*}{$\mathrm{MuA}$} & Aconitum angustifolium & E1 & . & . & . & . & . & . & . & + & . & 1 & 11 \\
\hline & Differential species of the subassociations (Razlikovalne vrste subase & iacij) & & & & & & & & & & & \\
\hline MA & Deschampsia cespitosa & E1 & 2 & 3 & 2 & 3 & 1 & 2 & . & . & . & 6 & 67 \\
\hline FS & Myosotis sylvatica agg. & E1 & 1 & + & 1 & + & + & + & . & . & . & 6 & 67 \\
\hline VP & Lonicera nigra & $\mathrm{E} 2 \mathrm{a}$ & + & 1 & + & + & + & + & . & . & . & 6 & 67 \\
\hline $\mathrm{MuA}$ & Primula elatior & E1 & + & + & + & + & . & . & . & . & . & 4 & 44 \\
\hline VP & Calamagrostis arundinacea & E1 & + & + & . & . & 1 & 1 & . & . & . & 4 & 44 \\
\hline TR & Molopospermum peloponnesiacum subsp. bauhinii & E1 & . & + & . & . & + & + & . & . & . & 3 & 33 \\
\hline
\end{tabular}


Number of relevé (Zaporedna številka popisa)

EP Calamagrostis varia

ES Horminum pyrenaicum

EP Rhodothamnus chamaecistus

VP Vaccinio-Piceetea

Vaccinium myrtillus

Dryopteris dilatata

Dryopteris expansa

Homogyne alpina

Luzula sylvatica

Oxalis acetosella

Phegopteris connectilis

Polystichum lonchitis

Gymnocarpium dryopteris

Maianthemum bifolium

Vaccinium vitis-idaea

Gentiana asclepiadea

Solidago virgaurea

Lonicera caerulea

Rosa pendulina

Clematis alpina

Picea abies

Picea abies

Picea abies

Luzula pilosa

Valeriana tripteris

Calamagrostis villosa

Luzula luzulina

Luzula luzuloides

Saxifraga cuneifolia

Huperzia selago

EP Erico-Pinetea

Rubus saxatilis

Cirsium erisithales

Erica carnea

Carex ornithopoda

SSC Sambuco-Salicion capreae

Rubus idaeus

Fragaria vesca

Urtica dioica

AF Aremonio-Fagion

Cardamine enneaphyllos

Cardamine trifolia

Cyclamen purpurascens

FS Fagetalia sylvaticae

Dryopteris filix-mas

Paris quadrifolia

Prenanthes purpurea

Acer pseudoplatanus

Acer pseudoplatanus

Acer pseudoplatanus

Petasites albus

Epilobium montanum

Melica nutans

\begin{tabular}{|c|c|c|c|c|c|c|c|c|c|c|c|}
\hline E1 & . & . & & . & . & r & 1 & 1 & + & 3 & 33 \\
\hline E1 & 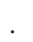 & . & & . & & & + & 1 & + & & 33 \\
\hline E1 & . & . & & . & r & · & . & + & + & 2 & 22 \\
\hline E1 & 1 & 1 & + & 1 & 2 & 1 & 4 & 3 & ? & 9 & 100 \\
\hline E1 & 2 & 2 & 2 & 2 & 2 & 1 & 1 & 1 & 1 & 9 & 100 \\
\hline E1 & + & . & . & + & + & + & 2 & 2 & 2 & 7 & 78 \\
\hline E1 & + & 1 & + & + & . & 1 & 1 & 1 & & 8 & 89 \\
\hline E1 & 1 & 3 & 2 & 2 & 2 & 1 & r. & . & + & 7 & 78 \\
\hline E1 & 1 & 1 & 1 & 1 & + & 1 & . & . & 1 & 7 & 78 \\
\hline E1 & + & + & + & + & . & 1 & . & + & + & 7 & 78 \\
\hline E1 & + & . & . & + & + & + & + & 1 & 1 & 7 & 78 \\
\hline E1 & . & + & + & + & + & 1 & . & + & + & 7 & 78 \\
\hline E1 & . & 1 & + & + & + & + & + & . & + & 7 & 78 \\
\hline E1 & . & + & + & + & + & 1 & 1 & . & 1 & 7 & 78 \\
\hline E1 & + & + & + & . & + & + & . & + & . & 6 & 67 \\
\hline E1 & . & + & + & + & 1 & 1 & . & + & . & 6 & 67 \\
\hline $\mathrm{E} 2 \mathrm{a}$ & . & + & + & 1 & + & + & . & . & r & 6 & 67 \\
\hline $\mathrm{E} 2 \mathrm{a}$ & + & . & . & + & . & . & 1 & . & + & 4 & 44 \\
\hline E2a & . & . & . & + & . & 1 & + & + & $\cdot$ & 4 & 44 \\
\hline E3 & . & . & . & . & $\mathrm{r}$ & + & . & $\mathrm{r}$ & r & 4 & 44 \\
\hline $\mathrm{E} 2 \mathrm{~b}$ & . & . & . & . & . & + & . & . & . & 1 & 11 \\
\hline E2a & . & . & . & . & + & . & . & $\mathrm{r}$ & . & 2 & 22 \\
\hline E1 & . & . & . & . & . & + & + & + & + & 4 & 44 \\
\hline E1 & + & . & . & . & . & + & . & . & + & 3 & 33 \\
\hline E1 & . & . & + & . & . & . & . & + & + & 3 & 33 \\
\hline E1 & . & . & . & . & . & + & . & + & + & 3 & 33 \\
\hline E1 & . & . & . & . & . & + & . & . & + & 2 & 22 \\
\hline E1 & . & . & . & . & . & + & . & . & + & 2 & 22 \\
\hline E1 & . & . & . & + & . & . & . & . & . & 1 & 11 \\
\hline E1 & + & . & . & + & . & 1 & 2 & 2 & 1 & 6 & 67 \\
\hline E1 & + & + & . & . & . & + & . & . & + & 4 & 44 \\
\hline E1 & . & . & . & . & . & + & + & + & + & 4 & 44 \\
\hline E1 & . & . & . & . & . & . & . & . & + & 1 & 1 \\
\hline E2a & 3 & 3 & 3 & 3 & 3 & 1 & . & 1 & + & 8 & 89 \\
\hline E1 & + & 1 & . & + & + & + & . & . & + & 6 & 67 \\
\hline E1 & . & . & + & . & . & . & . & + & . & 2 & 22 \\
\hline E1 & + & + & $\cdot$ & + & + & + & . & . & + & 6 & 67 \\
\hline E1 & . & . & . & . & 2 & . & . & . & 2 & 2 & 22 \\
\hline E1 & . & . & . & . & . & . & + & . & . & 1 & 1 \\
\hline E1 & + & + & + & + & + & 1 & + & + & + & 9 & 100 \\
\hline E1 & + & . & + & + & + & + & 1 & 1 & 1 & 8 & 89 \\
\hline E1 & + & + & + & . & . & . & + & . & + & 5 & 56 \\
\hline E3 & + & . & . & $\mathrm{r}$ & . & . & . & . & . & 2 & \\
\hline E2 & + & + & . & + & . & . & + & + & . & 5 & 56 \\
\hline E1 & . & 1 & . & . & . & . & . & . & . & 1 & 11 \\
\hline E1 & + & + & . & + & . & + & . & . & + & 5 & 56 \\
\hline E1 & . & 1 & + & . & + & + & . & + & . & 5 & \\
\hline E1 & . & . & . & . & . & + & + & 1 & 1 & 4 & \\
\hline
\end{tabular}


Number of relevé (Zaporedna števillka popisa)

Galeobdolon flavidum

Lonicera alpigena

Lathyrus vernus subsp. vernus

Galium laevigatum

Lilium martagon

Polystichum aculeatum

Actaea spicata

Fagus sylvatica

Fagus sylvatica

Luzula nivea

Poa nemoralis

Festuca heterophylla

QF Querco-Fagetea

Anemone nemorosa

Sorbus aria

QP Sorbus aria

QP Sorbus aria

BA Betulo-Alnetea

Salix appendiculata

Salix appendiculata

Salix appendiculata

Sorbus chamaemespilus

MuA Mulgedio-Aconitetea

Athyrium filix-femina

Polygonatum verticillatum

Veratrum album

Chaerophyllum hirsutum

Senecio cacaliaster

Viola biflora

Aconitum lycoctonum subsp. ranunculifolium (A. lupicida?)

Saxifraga rotundifolia

Senecio ovatus

Stellaria nemorum

Ranunculus platanifolius

Geum rivale

Rumex arifolius

Hypericum maculatum

Athyrium distentifolium

Allium victorialis

Grafia golaka

Thalictrum aquilegiifolium

\section{E1}

E2a

E1

E1

E1

E1

E1

E2

E1

E1

E1

E1

$\begin{array}{llllllllllllll}\mathrm{E} 1 & . & . & . & . & . & & 1 & 1 & 1 & 3 & 33\end{array}$

E3 $\mathrm{r}+$. . . . . . . 22

$\mathrm{E} 2 \mathrm{~b}$. $. \quad . \quad . \quad . \quad . \quad . \quad+\quad+11$

$\mathrm{E} 2 \mathrm{a} . \quad . \quad . \quad . \quad . \quad . \quad+\quad+11$

E3a r . + . . + . +4444

$\mathrm{E} 2 \mathrm{~b}++1++1 .+189$

$\mathrm{E} 2 \mathrm{a} . .5 . .+1 .+11$

$\mathrm{E} 2+.++++167$

$\begin{array}{llllllllllll}\mathrm{E} 1 & 2 & 2 & 1 & 1 & 2 & 1 & 1 & 1 & 2 & 9 & 100\end{array}$

$\mathrm{E} 1222+\ldots+11+89$

$\mathrm{E} 1+++++\quad 1 \quad 1 \quad 1 \quad 8 \quad 89$

$\mathrm{E} 12121+1.1+89$

$\mathrm{E} 1++1++.+++78$

$\mathrm{E} 1++\ldots++\ldots+167$

$\mathrm{E} 1 .++++{ }_{+}+667$

$\mathrm{E} 1+++\ldots+.556$

$\mathrm{E} 1+++\ldots+.556$

$\mathrm{E} 11+++. .2 . \quad . \quad 444$

$\mathrm{E} 1+.+++.+2.444$

$\mathrm{E} 1+.++.+4 .+44$

$\mathrm{E} 1+.+.+2 .+33$

$\mathrm{E} 1 .+.+2 .+2 .+33$

$\mathrm{E} 1 . \quad . \quad 1 \quad . \quad . \quad . \quad . \quad . \quad 1 \quad 11$

$\mathrm{E} 1$. . . . + . . . . . 111

$\mathrm{E} 1$. . . . + . . . . 111

$\mathrm{E} 1$. . . . . + . . . . 111

ES Elyno-Seslerietea

Betonica alopecuros

Sesleria caerulea subsp. calcaria

Campanula witasekiana

Soldanella alpina

Carex ferruginea

Aster bellidiastrum

Koeleria eriostachya

Festuca calva

Koeleria pyramidata

Helianthemum nummularium subsp. grandiflorum

Laserpitium peucedanoides

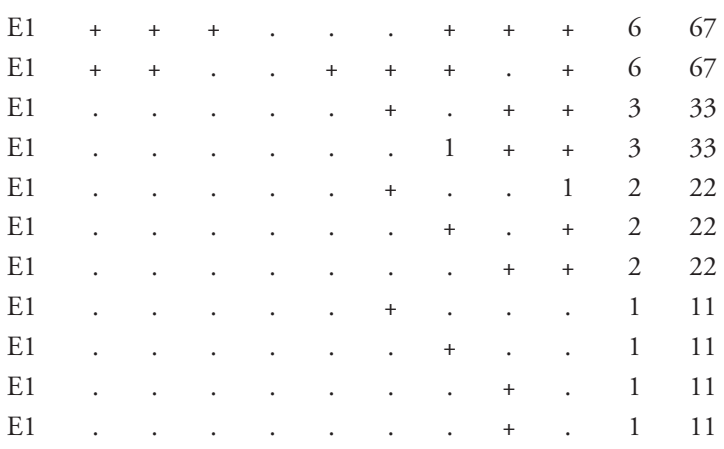


Alchemilla alpigena

Astrantia bavarica

Carex firma

Carex sempervirens

Galium anisophyllum

CU Calluno-Ulicetea

Potentilla erecta

Gentiana pannonica

Anthoxanthum odoratum

Agrostis capillaris

MA Molinio-Arrhenatheretea

Taraxacum officinale

Festuca rubra

SCF Scheuchzerio-Caricetea fuscae

Parnassia palustris

Selaginella selaginoides

TR Thlaspietea rotundifolii

Adenostyles glabra

Festuca nitida

Campanula cespitosa

Dryopteris villarii

AT Asplenietea trichomanis

Asplenium viride

Cystopteris fragilis

O Other species (Druge vrste)

Alchemilla sp.

ML Mosses and lichens (Mahovi in lišaji)

Dicranum scoparium

Ctenidium molluscum

Rhytidiadelphus triquetrus

Polytrichum formosum

Tortella tortuosa

Rhytidiadelphus loreus

Fissidens dubius

Plagiothecium undulatum

Marchantia polymorpha

Mnium thomsonii

Peltigera leucophlebia

Rhizomnium punctatum

Bryum capillare

Cladonia pyxidata
E1

E

E1

E1

E1

E1

E1

E1

E1

E1

E1 . . . . . . . + + . 111

E1 . . . . . . . . . +111

$\mathrm{E} 1 .+.+{ }^{2}+++444$

$\mathrm{E} 1+1+3+33$

$\mathrm{E} 1 . .5 . \quad . \quad . \quad 111$

E1 . . . . . . . . . 111

$\mathrm{E} 1 .+2 .+1+1156$

$\mathrm{E} 1 .+.+. \quad . \quad . \quad 111$

$\mathrm{E} 1$. . . . . $\quad+\quad . \quad . \quad 111$

$\mathrm{E} 0++.+++4.78$

$\mathrm{E} 0++.+1+1+167$

$\mathrm{E} 01 .+1+\ldots+67$

$\mathrm{E} 0++.+++.+667$

$\mathrm{E} 0++.+5+56$

$\mathrm{E} 01+++. \quad . \quad . \quad 444$

$\mathrm{E} 0$. . . . ++++444

$\mathrm{E} 0$. . . + . . . . 222

$\mathrm{E} 0$. . . . . +22

$\mathrm{E} 0$. . ..$+. . \quad . \quad 111$

$\mathrm{E} 0$. . . . . . . . . 111

$\mathrm{E} 0$. . . . . + . . . . 111

$\mathrm{E} 0$. . . . . + . . . . 111

$\mathrm{E} 0$. . . . . . . +11

\section{Legend - Legenda}

A Limestone - apnenec

Re Rendzina - rendzina 
Table 2: Synoptic table of syntaxa Alno viridis-Sorbetum aucupariae and Rhododendro hirsuti-Sorbetum aucupariae.

Tabela 2: Sintezna preglednica sintaksonov Alno viridis-Sorbetum aucupariae in Rhododendro hirsuti-Sorbetum aucupariae.

\section{Successive number (Zaporedna številka)}

Sign for syntaxa (Oznaka sintakonov)

Number of relevé (Število popisov)

\begin{tabular}{llll}
3 & 7 & 6 & 3 \\
\hline
\end{tabular}

Diagnostic species of the association Alno viridis-Sorbetum aucupariae (Diagnostične vrste asociacije)

BA Alnus viridis

MA Veratrum album subsp. lobelianum

MA Senecio cacaliaster

BA Salix appendiculata

BA Salix appendiculata

BA Salix appendiculata

Diagnostic species of the association Rhododendro hirsuti-Sorbetum aucupariae (Diagnostične vrste asociacije)

EP Pinus mugo

EP Rhododendron hirsutum

BA Salix waldsteiniana

VP Lycopodium annotinum

Differential species of the subassociations (Razlikovalnice subasociacij)

VP Luzula sylvatica

BA Sorbus chamaemespilus

$\mathrm{CU}$ Carex pilulifera

TR Adenostyles glabra

AT Asplenium viride

MA Angelica sylvestris

AT Cystopteris fragilis

JT Soldanella alpina

MA Deschampsia cespitosa

FS Myosotis sylvatica agg.

VP Lonicera nigra

VP Calamagrostis arundinacea

MuA Primula elatior

TR Molopospermum peloponnesiacum subsp. bauhinii

ES Horminium pyrenaicum

EP Calamagrostis varia

EP Rhodothamnus chamaecistus

SSC Sambuco-Salicion capreae

Sorbus aucuparia

Sorbus aucuparia

Sorbus aucuparia

Sorbus aucuparia

Rubus idaeus

Salix caprea

Salix caprea

Fragaria vesca

Urtica dioica

MuA Mulgedio-Aconitetea

Athyrium filix-femina

Polygonatum verticillatum

Saxifraga rotundifolia

Ranunculus platanifolius

Rumex arifolius

Adenostyles alliariae

Streptopus amplexifolius

Athyrium distentifolium

Stellaria nemorum

Allium victorialis

\begin{tabular}{|c|c|c|c|c|}
\hline E2b & 100 & 86 & & \\
\hline $\mathrm{F} 1$ & 100 & 100 & 83 & 100 \\
\hline & 67 & 100 & 83 & 67 \\
\hline & . & . & 50 & . \\
\hline & 33 & 100 & 100 & 67 \\
\hline & 33 & 57 & & 33 \\
\hline
\end{tabular}

\begin{tabular}{llc|cc|} 
E2 & 33 & $\cdot$ & 100 & 100 \\
E2a & $\cdot$ & 29 & 67 & 100 \\
E2a & $\cdot$ & 29 & 83 & 67 \\
E1 & $\cdot$ & $\cdot$ & 100 & 100 \\
\cline { 3 - 4 }
\end{tabular}

$\begin{array}{lllll}\mathrm{E} 1 & 100 & 14 & 100 & 33\end{array}$

E2 $100 \quad . \quad 50 \quad 100$

E1

E1

E1

E1

E1

E1

E1

E1

E1

E1

$\begin{array}{lllll}\text { E3 } & 100 & 100 & 100 & 100\end{array}$

$\begin{array}{lllll}\text { E2b } & 33 & 29 & 67 & 100\end{array}$

$\begin{array}{lllll}\text { E2a } & 100 & 57 & 67 & 67\end{array}$

$\begin{array}{lllll}\text { E1 } & 33 & 29 & 17 & 67\end{array}$

$\begin{array}{lllll}\text { E2a } & 100 & 100 & 100 & 67\end{array}$

E3 33 . .

E2b 33 . .

$\begin{array}{lllll}\text { E1 } & 43 & 83 & 33\end{array}$

$\begin{array}{llll}\text { E1 } & 29 & 17 & 33\end{array}$

$\begin{array}{lllll}\text { E1 } & 100 & 100 & 100 & 100\end{array}$

$\begin{array}{lllll}\mathrm{E} 1 & 100 & 86 & 83 & 100\end{array}$

$\begin{array}{lllll}\text { E1 } & 33 & 71 & 67 & 33\end{array}$

$\begin{array}{lllll}\mathrm{E} 1 & 33 & 86 & 67\end{array}$

$\begin{array}{llll}\text { E1 } & 33 & 57 & 50\end{array}$.

E1 $33 \quad 43$. .

E1 $33 \quad 14 \quad$.

$\begin{array}{llll}\text { E1 } & 33 & 14 & 17 .\end{array}$

E1 $33 \quad 14 \quad 67$.

E1 33 . 17 . 
Successive number (Zaporedna številka)

Millium effusum

Geum rivale

Thalictrum aquilegiifolium

Aconitum lycoctonum s. lat. (A. lupicida)

Geranium sylvaticum

Chaerophyllum hirsutum

Viola biflora

Alchemilla xanthochlora

Hypericum maculatum

Pleurospermum austriacum

Heracleum montanum (inc. H. pollinianum)

Phyteuma ovatum

Myrrhis odorata

Doronicum austriacum

Lathyrus occidentalis var. montanus

Senecio ovatus

Aconitum degenii subsp. paniculatum

Agropyron caninum

Crepis paludosa

Poa hybrida

Senecio rivularis

Silene dioica

Chaerophyllum aureum

Tanacetum corymbosum subsp. clusii

Grafia golaka

VP Vaccinio-Piceetea

Dryopteris dilatata

Gentiana asclepiadea

Oxalis acetosella

Rosa pendulina

Maianthemum bifolium

Vaccinium myrtillus

Phegopteris connectilis

Luzula luzuloides subsp. luzuloides

Picea abies

Picea abies

Picea abies

Blechnum spicant

Solidago virgaurea

Veronica urticifolia

Luzula luzulina

Thelypteris limbosperma

Luzula luzuloides subsp. rubella

Valeriana tripteris

Polystichum lonchitis

Dryopteris expansa

Homogyne alpina

Gymnocarpium dryopteris

Lonicera caerulea

Saxifraga cuneifolia

Abies alba

Homogyne sylvestris

Pyrola minor

Vacinium vitis-idaea

Clematis alpina

Luzula pilosa

Calamagrostis villosa

Huperzia selago

E1

E1

E1

$\begin{array}{lll}86 & 50 \quad 100\end{array}$

E1 . 86.

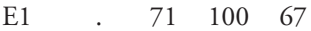

E1 . $\quad \begin{array}{lll}71 & 50 & 100\end{array}$

E1 . 71.

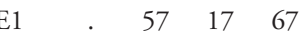

E1 . 57.

E1. $43 .$.

E1. 43.

E1. 43. .

E1 . 29. .

E1 . 29.

$\begin{array}{lllll}\text { E1 } & 14 & 67 & 33\end{array}$

E1. 14 .

E1 . 14. .

E1 . 14. .

E1 . 14.

E1. 14.

E1. 14 .

E1 . 14.

E1 . 14.

E1 . . 17 .

$\begin{array}{lllll}\mathrm{E} 1 & 100 & 57 & 100 & 100\end{array}$

$\begin{array}{lllll}\text { E1 } & 67 & 86 & 83 & 33\end{array}$

$\begin{array}{lllll}\text { E1 } & 67 & 86 & 100 & 33\end{array}$

$\begin{array}{lllll}\text { E2a } & 67 & 86 & 33 & 67\end{array}$

$\begin{array}{lllll}\text { E1 } & 67 & 29 & 83 & 67\end{array}$

$\begin{array}{lllll}\text { E1 } & 67 & 29 & 100 & 100\end{array}$

$\begin{array}{lllll}\text { E1 } & 67 & 29 & 83 & 67\end{array}$

$\begin{array}{lllll}\text { E1 } & 67 & 14 & 17 & 33\end{array}$

$\begin{array}{lllll}\text { E3 } & 67 & 33 & 67\end{array}$

$\begin{array}{llll}\text { E2b } & 67 & 14 & 17\end{array}$

$\begin{array}{lllll}\text { E2a } & 67 & 14 & . & 33\end{array}$

E1 $67 \quad . \quad$.

$\begin{array}{lllll}\text { E1 } & 33 & 86 & 83 & 33\end{array}$

E1 $33 \quad 14$.

$\begin{array}{llll}\mathrm{E} 1 & 33 & 17 & 67\end{array}$

E1 33 . .

E1 33.53

$\begin{array}{lllll}\mathrm{E} 1 & . & 86 & 33 & 33\end{array}$

$\begin{array}{lllll}\mathrm{E} 1 & 57 & 67 & 100\end{array}$

$\begin{array}{lllll}\text { E1 } & . & 29 & 67 & 100\end{array}$

$\begin{array}{lllll}\text { E1 . } & 14 & 83 & 100\end{array}$

$\begin{array}{lllll}\mathrm{E} 1 & . & 14 & 83 & 67\end{array}$

E2a . $\quad \begin{array}{lll}14 & 83 \quad 33\end{array}$

E1 . $14 \quad 17 \quad 33$

E1 . 14.

E1 . 14

E1 . 14.

E1 . . $\quad 83 \quad 67$

E1 . . $33 \quad 67$

E1 . . $\quad 17 \quad 100$

E1 . . $17 \quad 67$

E1 . 17. 
EP Erico-Pinetea

Rubus saxatilis

Cirsium erisithales

Carex ornithopoda

Molinia caerulea subsp. arundinacea

Aquilegia nigricans

Erica carnea

AF Aremonio-Fagion

Cardamine trifolia

Knautia drymeia

Rhamnus fallax

Cardamine enneaphyllos

Anemone trifolia

Cyclamen purpurascens

TA Tilio-Acerion

Acer pseudoplatanus

Acer pseudoplatanus

Acer pseudoplatanus

Polystichum braunii

Polystichum $x$ luerssenii

Hesperis candida

Lunaria rediviva

Adoxa moschatellina

FS Fagetalia sylvaticae

Fagus sylvatica

Fagus sylvatica

Fagus sylvatica

Paris quadrifolia

Prenanthes purpurea

Dryopteris filix-mas

Galium laevigatum

Lilium martagon

Laburnum alpinum

Laburnum alpinum

Laburnum alpinum

Scrophularia nodosa

Polystichum aculeatum

Symphytum tuberosum

Actaea spicata

Lathyrus vernus subsp. vernus

Epilobium montanum

Daphne mezereum

Phyllitis scolopendrium

Aruncus dioicus

Galeobdolon flavidum

Lonicera alpigena

Luzula nivea

Mercurialis perennis

Carex sylvatica

Ranunculus lanuginosus

Campanula trachelium

Lathyrus vernus subsp. flaccidus

Melica nutans

Petasites albus

Poa nemoralis

Festuca heterophylla

QF Querco-Fageted

Anemone nemorosa

\begin{tabular}{|c|c|c|c|c|}
\hline 1 & 33 & 86 & 50 & 100 \\
\hline E1 & & 43 & 50 & 33 \\
\hline 1 & & 29 & & 33 \\
\hline $\mathrm{E} 1$ & . & 29 & & \\
\hline 1 & & 14 & & \\
\hline E1 & . & & 17 & 100 \\
\hline & 33 & . & 17 & 33 \\
\hline E1 & . & 14 & & \\
\hline $2 b$ & . & 14 & & \\
\hline E1 & . & 14 & 83 & 33 \\
\hline 1 & . & . & 67 & \\
\hline 1 & . & & & 33 \\
\hline E3 & 67 & 57 & 33 & \\
\hline E2 & . & 14 & 50 & 67 \\
\hline E1 & 67 & 43 & 17 & \\
\hline E1 & 33 & . & 33 & \\
\hline E1 & . & . & 33 & \\
\hline E1 & & 14 & . & \\
\hline $\mathrm{E} 1$ & . & 14 & . & \\
\hline E1 & . & 14 & 17 & \\
\hline E3 & 33 & & . & \\
\hline E2 & 67 & & 17 & \\
\hline E1 & 33 & 14 & 17 & \\
\hline E1 & 67 & 100 & 83 & 100 \\
\hline E1 & 67 & . & 50 & 67 \\
\hline E1 & 33 & 100 & 100 & 100 \\
\hline E1 & 33 & 71 & 17 & \\
\hline E1 & 33 & 71 & 17 & \\
\hline E3 & 33 & 29 & 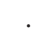 & \\
\hline $\mathrm{E} 2 \mathrm{a}$ & . & 14 & . & \\
\hline E1 & . & 14 & . & \\
\hline E1 & 33 & 14 & . & \\
\hline E1 & . & 57 & 17 & \\
\hline E1 & . & 57 & . & \\
\hline E1 & . & 43 & 17 & \\
\hline E1 & 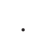 & 43 & 33 & . \\
\hline E1 & - & 29 & 67 & 33 \\
\hline $\mathrm{E} 2 \mathrm{a}$ & . & 29 & . & 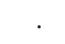 \\
\hline E1 & 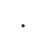 & 29 & . & \\
\hline E1 & & 29 & . & \\
\hline E1 & . & 14 & 17 & 67 \\
\hline E2a & . & 14 & 50 & . \\
\hline E1 & 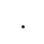 & 14 & 17 & - \\
\hline E1 & - & 14 & . & \\
\hline E1 & & 14 & & \\
\hline E1 & . & 14 & 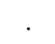 & \\
\hline E1 & r & 14 & & \\
\hline E1 & 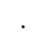 & 14 & . & \\
\hline E1 & $\cdot$ & . & 17 & 100 \\
\hline E1 & & & 67 & 33 \\
\hline E1 & - & - & 17 & . \\
\hline E1 & . & - & . & 33 \\
\hline & 67 & 86 & & 10 \\
\hline
\end{tabular}


Successive number (Zaporedna številka)

QP Sorbus aria

QP Sorbus aria

Dactylorhiza fuchsii

ES Elyno-Seslerietea

Sesleria caerulea subsp. calcaria

Campanula witasekiana

Festuca calva

Hieracium pilosum

Carex ferruginea

Betonica alopecuros

Aster bellidiastrum

Carex sempervirens

FB Koeleria pyramidata

Heliosperma alpestre

Koelerya eryostachia

Helianthemum grandiflorum

Laserpitium peucedanoides

Astrantia bavarica

Carex firma

Galium anisophyllum

SC Schenchzerio-Caricetea fuscae

Parnassia palustris

Selaginella selaginoides

$\mathrm{CU}$ Calluno-Ulicetea

Anthoxanthum odoratum

Gentiana pannonica

$\mathrm{PaT}$ Poo alpinae-Trisetetalia

Poa alpina

Trollius europaeus

Pimpinella major subsp. rubra

Potentilla erecta

Agrostis capillaris

MA Molinio-Arrhenatheretea

Taraxacum officinale

Festuca rubra

TR Thlaspietea rotundifolii

Alchemilla alpigena

Rhodiola rosea

Festuca nitida

Dryopteris villarii

Arabis alpina

Cystopteris montana

Hieracium bifidum

Campanula cespitosa

AT Asplenietea trichomanis

Paederota lutea

Campanula carnica

Asplenium trichomanes

Sedum maximum

Festuca stenantha

Primula auricula

Asplenium ruta-muraria

Cerastium subtriflorum

Saxifraga crustata

$\mathrm{O}$ Other species (Druge viste)

Alchemilla sp.

ML Mosses and lichens (Mahovi in lišaji)

Polytrichum formosum

\begin{tabular}{|c|c|c|c|c|}
\hline E3 & & & 33 & \\
\hline $\mathrm{E} 2 \mathrm{~b}$ & 67 & 14 & . & 33 \\
\hline $\mathrm{E} 1$ & . & 43 & . & . \\
\hline E1 & . & 71 & 67 & 67 \\
\hline E1 & . & 29 & 17 & 67 \\
\hline E1 & . & 29 & 17 & . \\
\hline E1 & . & 29 & . & . \\
\hline E1 & . & 29 & 17 & 33 \\
\hline E1 & . & 14 & 50 & 100 \\
\hline E1 & . & 14 & . & 67 \\
\hline E1 & . & 14 & . & 33 \\
\hline E1 & . & 14 & . & 33 \\
\hline E1 & . & 14 & . & . \\
\hline E1 & . & . & . & 67 \\
\hline E1 & . & . & . & 33 \\
\hline E1 & . & . & . & 33 \\
\hline E1 & . & . & . & 33 \\
\hline E1 & . & . & . & 33 \\
\hline E1 & . & . & . & 33 \\
\hline E1 & . & . & . & 33 \\
\hline E1 & . & . & . & 33 \\
\hline E1 & . & . & 17 & . \\
\hline E1 & 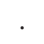 & . & 17 & . \\
\hline E1 & . & 43 & . & . \\
\hline E1 & . & 43 & . & \\
\hline E1 & . & 29 & . & . \\
\hline E1 & . & . & . & 33 \\
\hline E1 & . & . & . & 33 \\
\hline E1 & . & 14 & 17 & \\
\hline E1 & . & . & . & 33 \\
\hline E1 & . & 57 & . & 33 \\
\hline E1 & . & 57 & . & . \\
\hline E1 & . & 43 & . & 100 \\
\hline E1 & . & 29 & . & 33 \\
\hline E1 & . & 14 & . & . \\
\hline E1 & . & 14 & . & . \\
\hline E1 & . & 14 & . & . \\
\hline E1 & . & . & . & 33 \\
\hline E1 & . & 57 & . & . \\
\hline E1 & . & 29 & . & . \\
\hline E1 & . & 29 & . & \\
\hline E1 & . & 14 & . & . \\
\hline E1 & . & 14 & . & \\
\hline E1 & . & 14 & . & . \\
\hline E1 & . & 14 & . & . \\
\hline E1 & . & 14 & . & \\
\hline E1 & . & 14 & . & . \\
\hline E1 & . & 14 & 17 & . \\
\hline E0 & 67 & 43 & 67 & 67 \\
\hline
\end{tabular}


Successive number (Zaporedna številka)

Rhytidiadelphus triquetrus

Eurhynchium striatum

Pseudoleskeella catenulata

Ctenidium molluscum

Tortella tortuosa

Isothecium alopecuroides

Schistidium apocarpum

Mnium thomsonii

Hylocomium splendens

Rhytidiadelphus loreus

Cladonia pyxidata

Dicranum scoparium

Marchantia polymorpha

Peltigera canina

Atrichum undulatum

Fisidens dubius

Plagiothecium undulatum

Peltigera leucophlebia

Rhizomnium punctatum

Bryum capillare

\begin{tabular}{|c|c|c|c|c|}
\hline & 1 & 2 & 3 & 4 \\
\hline E0 & 33 & 86 & 83 & 33 \\
\hline E0 & 33 & . & . & . \\
\hline E0 & 33 & . & . & . \\
\hline E0 & . & 100 & 50 & 100 \\
\hline E0 & . & 43 & 50 & 67 \\
\hline E0 & . & 29 & . & . \\
\hline E0 & . & 29 & . & . \\
\hline E0 & . & 29 & 17 & . \\
\hline E0 & . & 14 & . & . \\
\hline E0 & . & 14 & 67 & . \\
\hline E0 & . & 14 & . & 33 \\
\hline E0 & . & 14 & 83 & 67 \\
\hline E0 & . & 14 & 17 & 33 \\
\hline E0 & . & 14 & . & . \\
\hline E0 & . & 14 & . & . \\
\hline E0 & . & . & 17 & 100 \\
\hline E0 & . & . & 33 & . \\
\hline E0 & . & . & 17 & . \\
\hline E0 & . & . & 17 & . \\
\hline E0 & . & . & 17 & \\
\hline
\end{tabular}

\section{Legend - Legenada}

AvSals Alno viridis-Sorbetum aucupariae luzuletosum sylvaticae

AvSaad Alno viridis-Sorbetum aucupariae adenostyletosum glabrae

RhSadc Rhododendro hirsuti-Sorbetum aucupariae deschampsietosum cespitosae

RhSacv Rhododendro hirsuti-Sorbetum aucupariae calamagrostietosum variae

Table 3: Phytosciological groups of the associations Alno viridis-Sorbetum aucupariae and Rhododendro hirsuti-Sorbetum aucupariae.

Tabela 3: Phytosciological groups of the associations Alno viridis-Sorbetum aucupariae in Rhododendro hirsuti-Sorbetum aucupariae.

\begin{tabular}{lcccc} 
Successive number (Zaporedna številka) & 1 & 2 & 3 & 4 \\
Sign for syntaxa (Oznaka sintakonov) & AvSals & AvSaad & RhSadc & RhSacv \\
Number of relevé (Število popisov) & 3 & 7 & 6 & 3 \\
\hline Sambuco-Salicetum capreae & 10.5 & 5.4 & 6.9 & 6.4 \\
Betulo-Alnetea & 6.5 & 3.8 & 4.3 & 3.7 \\
Mulgedio-Aconitetea & 16.2 & $\mathbf{2 3 . 8}$ & $\mathbf{1 6 . 3}$ & 11.0 \\
Vaccinio-Piceetea & $\mathbf{2 9 . 4}$ & 13.0 & $\mathbf{2 6 . 2}$ & 22.0 \\
Erico-Pinetea & 1.6 & 3.2 & $\mathbf{4 . 3}$ & 8.7 \\
Aremonio-Fagion & 2.4 & 3.8 & 6.9 & 10.1 \\
Tilio-Acerion & 4.1 & 2.2 & 2.8 & 0.9 \\
Fagetalia sylvaticae & $\mathbf{1 9 . 5}$ & $\mathbf{1 6 . 4}$ & $\mathbf{1 6 . 6}$ & 9.2 \\
Querco-Fagetea & 3.3 & 2.0 & 0.5 & 1.8 \\
Elyno-Seslerietea. Scheuchzerio-Caricetea fuscae & 0 & 4.6 & 2.6 & $\mathbf{1 2 . 4}$ \\
Calluno-Ulicetea. Poo alpinae-Trisetetalia & 1.6 & 1.6 & 0.5 & 0.9 \\
Molinio-Arrhenatheretea & 0.8 & 2.8 & 1.8 & 0.5 \\
Thlaspietea rotundifolii & 0 & 5.6 & 1.0 & 4.1 \\
Asplenietea trichomanis & 0 & 5.4 & 0.8 & 0.3 \\
Other species (Druge vrste) & 0 & 0.2 & 6.4 & 6.9 \\
Mosses and lichens (Mahovi in lišaji) & 4.1 & 100.0 & 100 \\
Total (Skupaj) & 100 & & 0.3 \\
\hline
\end{tabular}

\section{Legend - Legenada}

AvSals Alno viridis-Sorbetum aucupariae luzuletosum sylvaticae

AvSaad Alno viridis-Sorbetum aucupariae adenostyletosum glabrae

RhSadc Rhododendro hirsuti-Sorbetum aucupariae deschampsietosum cespitosae

$\mathrm{RhSacv}$ Rhododendro hirsuti-Sorbetum aucupariae calamagrostietosum variae 
Table 4: Floristic composition and phytosociological structure of abandoned hay meadows under the Mt. Jalovnik.

Tabela 4: Floristična sestva in fitocenološka zgradba nekdanjih senožeti pod Jalovnikom.

\begin{tabular}{|c|c|c|c|c|c|c|c|c|c|c|}
\hline & Number of relevé (Zaporedna številka popisa) & & 1 & 2 & 3 & 4 & 5 & 6 & 7 & \\
\hline & Database number of relevé (Delovna številka popisa) & & 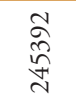 & $\begin{array}{l}\hat{\tilde{\alpha}} \\
\hat{n} \\
\approx\end{array}$ & $\underset{\stackrel{2}{\approx}}{\stackrel{\sim}{\sim}}$ & 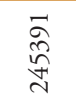 & 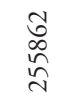 & $\begin{array}{l}\stackrel{8}{0} \\
\infty \\
\bumpeq \\
\sim\end{array}$ & $\begin{array}{l}\vec{\Xi} \\
\infty \\
\bumpeq \\
\end{array}$ & \\
\hline & Elevation in $\mathrm{m}$ (Nadmorska višina $\mathrm{v} \mathrm{m})$ & & 1400 & 1400 & 1375 & 1190 & 1150 & 1220 & 1090 & \\
\hline & Aspect (Lega) & & SE & SE & S & NEE & S & SE & SE & \\
\hline & Slope in degrees (Nagib v stopinjah) & & 10 & 25 & 40 & 35 & 30 & 30 & 25 & \\
\hline & Parent material (Matična podlaga) & & $\mathrm{R}$ & ALR & $\mathrm{R}$ & AR & ALR & AR & ALR & \\
\hline & Soil (Tla) & & Dy & $\mathrm{Eu}$ & Dy & $\mathrm{Eu}$ & $\mathrm{Eu}$ & $\operatorname{Re}$ & $\mathrm{Eu}$ & \\
\hline & Cover of shrub layer in \% (Zastiranje grmovne plasti v \%): & E2 & . & 20 & 5 & . & 10 & 5 & 5 & \\
\hline & Cover of herb layer in \% (Zastiranje zeliščne plasti v \%): & E1 & 100 & 90 & 95 & 100 & 100 & 100 & 100 & \\
\hline & Number of species (Število vrst) & & 21 & 28 & 20 & 44 & 57 & 72 & 76 & \\
\hline & Relevé area (Velikost popisne ploskve) & $\mathrm{m}^{2}$ & 20 & 40 & 20 & 20 & 40 & 40 & 40 & \\
\hline & Date of taking relevé (Datum popisa) & & 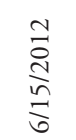 & 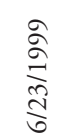 & 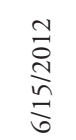 & $\frac{\stackrel{N}{a}}{\frac{i}{n}}$ & 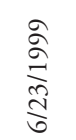 & 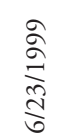 & 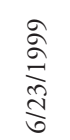 & \\
\hline & Quadrant (Kvadrant) & & $\begin{array}{l}\stackrel{N}{\infty} \\
\stackrel{\infty}{\infty} \\
\curvearrowright\end{array}$ & $\begin{array}{l}\frac{N}{\infty} \\
\stackrel{\infty}{\infty} \\
\stackrel{0}{\infty}\end{array}$ & $\begin{array}{l}\frac{N}{\infty} \\
\stackrel{\infty}{\infty} \\
\curvearrowright\end{array}$ & $\begin{array}{l}\frac{N}{\infty} \\
\stackrel{\infty}{\infty} \\
\infty\end{array}$ & $\begin{array}{l}\frac{N}{\infty} \\
\stackrel{+}{\infty} \\
\curvearrowright\end{array}$ & $\begin{array}{l}\frac{N}{\infty} \\
\stackrel{\infty}{\infty} \\
\curvearrowright\end{array}$ & $\begin{array}{l}\frac{N}{\infty} \\
\underset{\infty}{\infty} \\
\curvearrowright\end{array}$ & \\
\hline & Coordinate GK X (D-48) & $\mathrm{m}$ & 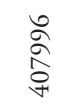 & $\begin{array}{l}\stackrel{+}{ } \\
\stackrel{2}{ } \\
\infty \\
\stackrel{+}{+}\end{array}$ & 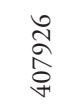 & 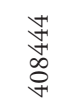 & \begin{tabular}{l}
$n$ \\
$\infty$ \\
$\infty$ \\
$\infty$ \\
\multirow{\gamma}{*}{}
\end{tabular} & \begin{tabular}{l}
$\hat{n}$ \\
$\hat{\alpha}$ \\
$\infty$ \\
$\&$ \\
\multirow{+}{*}{}
\end{tabular} & 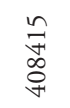 & \\
\hline & Coordinate GK X (D-48) & $\mathrm{m}$ & 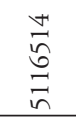 & 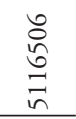 & 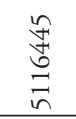 & $\begin{array}{l}\text { N } \\
\text { তర } \\
\stackrel{\Xi}{n}\end{array}$ & 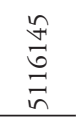 & 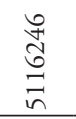 & $\begin{array}{l}\stackrel{m}{0} \\
\stackrel{0}{0} \\
\stackrel{\vec{n}}{n}\end{array}$ & \\
\hline & Diagnostic species of syntaxa (Diagnostične vrste sintaksonov) & & & & & & & & & Pr. \\
\hline $\mathrm{CU}$ & Nardus stricta & E1 & 2 & . & . & . & . & . & . & 1 \\
\hline VP & Homogyne alpina & E1 & 1 & . & . & . & . & . & . & 1 \\
\hline $\mathrm{CU}$ & Carex pallescens & E1 & + & + & . & . & . & . & . & 2 \\
\hline $\mathrm{CU}$ & Arnica montana & E1 & . & + & . & . & . & . & . & 1 \\
\hline EP & Molinia caerulea subsp. arundinacea & E1 & 4 & 2 & 3 & 4 & 1 & + & + & 7 \\
\hline $\mathrm{FB}$ & Iris sibirica subsp. erirrhiza & E1 & + & 1 & 4 & 2 & 1 & 1 & + & 7 \\
\hline $\mathrm{CU}$ & Calluno-Ulicetea & & & & & & & & & \\
\hline & Potentilla erecta & E1 & 1 & 1 & 1 & + & + & + & + & 7 \\
\hline & Phyteuma zahlbruckneri & E1 & . & . & . & . & 1 & . & + & 2 \\
\hline & Polygala vulgaris & E1 & . & . & . & . & . & . & + & 1 \\
\hline $\mathrm{FB}$ & Festuco-Brometea & & & & & & & & & \\
\hline & Cirsium erisithales & E1 & . & + & . & + & 1 & + & + & 5 \\
\hline & Carlina acaulis & E1 & . & + & . & . & + & + & + & 4 \\
\hline & Galium verum & E1 & . & . & . & + & 1 & 1 & 1 & 4 \\
\hline & Bromopsis transsilvanica & E1 & . & . & . & 1 & + & 1 & 1 & 4 \\
\hline & Euphorbia verrucosa & E1 & . & . & . & 1 & 1 & + & + & 4 \\
\hline & Salvia pratensis & E1 & . & . & . & + & + & + & 1 & 4 \\
\hline & Ranunculus polyanthemophyllus & E1 & . & . & . & + & + & + & + & 4 \\
\hline & Trifolium montanum & E1 & . & . & . & + & + & + & 1 & 4 \\
\hline & Koeleria pyramidata & E1 & . & . & . & + & . & 1 & 1 & 3 \\
\hline & Peucedanum oreoselinum & E1 & . & . & . & + & . & + & + & 3 \\
\hline & Buphthalmum salicifolium & E1 & . & . & . & + & . & 1 & 1 & 3 \\
\hline & Cirsium pannonicum & E1 & . & . & . & + & . & + & 1 & 3 \\
\hline & Cirsium x linkianum & E1 & . & . & . & + & . & + & + & 3 \\
\hline & Brachypodium rupestre & E1 & . & . & . & . & 1 & 1 & 1 & 3 \\
\hline & Gymnadenia conopsea & E1 & . & . & . & . & + & + & + & 3 \\
\hline & Helictotrichon praeustum & E1 & . & . & . & . & + & + & + & 3 \\
\hline
\end{tabular}


Hypochoeris maculata

Dianthus hysssopifolius

Briza media

Carex humilis

Genista tinctoria

Euphorbia cyparissias

Teucrium chamaedrys

Anthyllis vulneraria

Filipendula vulgaris

Stachys recta

Thymus pulegioides

PaT Poo alpinae-Trisetetalia

Astrantia major

Trollius europaeus

Traunsteinera globosa

Festuca nigrescens

Pimpinella major subsp. rubra

Anthoxanthum odoratum

Molinio-Arrhenatheretea

Vicia cracca

Lathyrus pratensis

E1

E1

E1

E1

E1

E1

E1

E1

E1

E1

E1

E1

E1

E1

E1

E1

E1

E1

E1

E1

Lotus corniculatus

Tragopogon orientalis

E1

E1

actylis glomerata

Galium album

Deschampsia cespitosa

Leontodon hispidus

Colchicum autumnale

Trifolium pratense

ES Elyno-Seslerietea

Betonica alopecuros

Carduus crassifolius

Centaurea haynaldii subsp. julica

Leucanthemum heterophyllum

Phyteuma orbiculare

Festuca calva

Biscutella laevigata

Phleum hirsutum

Sesleria caerulea subsp. calcaria

TG Trifolio-Geranietea

Verbascum lanatum

Geranium sanguineum

Achillea distans

Iris graminea

Thalictrum minus

Vicia sylvatica

Vincetoxicum hirundinaria

Valeriana collina ( $V$. wallrothii)

Inula birta

Lilium carniolicum

Polygonatum odoratum

Laserpitium latifolium

Trifolium medium
E1

E1

E1

E1

E1

E1

E1

E1

E1

E1

E1

E1

E1

E1

E1

E1

E1

E1

E1

E1

E1

E1

E1

E1

E1

E1

E1

$\begin{array}{lllllllll}1 & 2 & 3 & 4 & 5 & 6 & 7 & \text { Pr. }\end{array}$

1

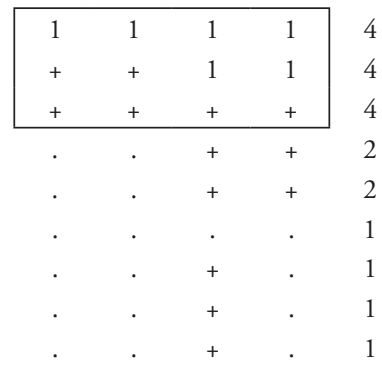

\begin{tabular}{|cccc|c}
\hline & + & + & + & 4 \\
1 & 1 & 1 & 1 & 4 \\
+ & 1 & 1 & 1 & 4 \\
1 & + & 1 & + & 4 \\
+ & 1 & 1 & + & 4 \\
+ & 1 & + & + & 4 \\
1 & + & 1 &. & 3 \\
+ & + & + &. & 3 \\
1 &. & 1 & + & 3 \\
$\cdot$ & + & 1 & + & 3 \\
$\cdot$ & 1 & 1 & $\cdot$ & 2 \\
$\cdot$ & + & + & $\cdot$ & 2 \\
$\cdot$ & 1 &. &. & 1
\end{tabular}


Silene nutans

Veronica teucrium

Hypericum perforatum

SSC Sambuco-Salicion capreae

Rubus idaeus

RP Rhamno-Prunetea

Juniperus communis

Rosa canina

Rosa glauca

Rosa villosa

Berberis vulgaris

MuA Mulgedio-Aconitetea, Betulo-Alnetea

Veratrum album subsp. lobelianum

Rumex arifolius

BA Sorbus chamaemespilus

Polygonatum verticillatum

Silene vulgaris subsp. antelopum

Geranium sylvaticum

Chaerophyllum aureum

Senecio ovatus

Hypericum maculatum

Aconitum lycoctonum

Aconitum angustifolium

FS Fagetalia sylvaticae

Knautia drymeia

Lilium martagon

QP Quercetalia pubescenti-petraeae

Sorbus aria

Convallaria majalis

Carex flacca

Tanacetum corymbosum

Mercurialis ovata

Cornus mas

QR Quercetalia roboris

Serratula tinctoria

Chamaecytisus supinus

Betonica officinalis

Hieracium sabaudum

Betula pendula

QF

Querco-Fagetea

Anemone nemorosa

Ornithogalum pyrenaicum

Veratrum nigrum

Carex montana

Platanthera bifolia

Cruciata glabra

Helleborus odorus

Primula vulgaris

Pyrus pyraster

VP

Vaccinio-Piceetea

Calamagrostis arundinacea

Luzula luzuloides subsp. rubella

Gentiana asclepiadea
E1

E1

E1

$\begin{array}{lllllll} & \mathrm{E} 2 \mathrm{a} & 2 & 2 & 1 & + & +\end{array}$

E2

E2

E2

E2a

E2a

E1 $111+1+1+1$

$\mathrm{E} 1+++.+4.4$

$\mathrm{E} 2+++.+2.3$

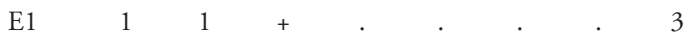

E1 . . . . $+t^{+}+3$

$\mathrm{E} 1++\quad+\quad . \quad$. $\quad .2$

$\mathrm{E} 1+\mathrm{E} . \quad . \quad+\quad+2$

$\mathrm{E} 1+. \quad . \quad$. $\quad \mathrm{r} \quad 2$

$\mathrm{E} 1+. \quad . \quad . \quad . \quad . \quad$. 1

E1

E1

E1

E1

E2

E1

E1

E1

E1

E2a

E1

E1

E1

E1

E2a

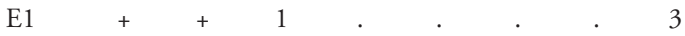

$\mathrm{E} 1$. $.6 .+2 .+2$

E1 . . . . . 18 r 1

$\mathrm{E} 1$. . . $\quad+\quad$. . . 1

$\mathrm{E} 1$. . . $\quad+\quad . \quad$. 1

E1 . . . . . .

E1 . . . . . . $5+$.

E1

E2a

E1 $111+2+6$

$\begin{array}{lllllll}\mathrm{E} 1 & 3 & 1 & 2 & + & + & +\end{array}$

$\begin{array}{llllllllllll}\text { E1 } & 1 & 1 & \mathrm{r} & . & . & & & & & & \end{array}$ 


\begin{tabular}{|c|c|c|c|c|c|c|c|c|c|}
\hline Number of relevé (Zaporedna številka popisa) & & 1 & 2 & 3 & 4 & 5 & 6 & 7 & Pr. \\
\hline Vaccinium myrtillus & E1 & 2 & + & + & . & . & . & . & 3 \\
\hline Avenella flexuosa & E1 & . & + & . & . & . & . & . & 1 \\
\hline Larix decidua & $\mathrm{E} 2 \mathrm{~b}$ & . & . & . & . & $\mathrm{r}$ & . & . & 1 \\
\hline Maianthemum bifolium & E1 & + & . & . & . & . & . & . & 1 \\
\hline Vaccinium vitis-idaea & E1 & + & . & . & . & . & . & . & 1 \\
\hline
\end{tabular}

\section{Legend - Legenada}

R Chert-roženec

A Limestone - apneenc

L Marl-lapor

Eu Eutryc brown soil - evtrična rjava tla

Dy Dystric brown soil - distrična rjava tla

Re Rendzina - rendzina
Relevé 1: Homogyno alpinae-Nardetum

Relevé 2: similar to Homogyno alpinae-Nardetum

Relevé 3: Molinio arundinaceae-Iridetum erirrhizae nom. prov. Relevé 4-7: Centaureo julici-Geranietum sanguinei nom. prov.

Table 5: Calamagrostio arundinaceae-Sorbetum ariae under Mts. Jalovnik and Krikov Vrh.

Tabela 5: Calamagrostio arundinaceae-Sorbetum ariae pod Jalovnikom in Krikovim vrhom.

\begin{tabular}{|c|c|c|c|c|c|c|}
\hline Number of relevé (Zaporedna številka popisa) & & 1 & 2 & 3 & 4 & 5 \\
\hline Database number of relevé (Delovna številka popisa) & & 245419 & 245423 & 245421 & 245422 & 257571 \\
\hline Elevation in $\mathrm{m}$ (Nadmorska višina $\mathrm{v} \mathrm{m})$ & & 1340 & 1150 & 1400 & 1330 & 1220 \\
\hline Aspect (Lega) & & SE & SE & $\mathrm{NE}$ & E & NW \\
\hline Slope in degrees (Nagib v stopinjah) & & 35 & 35 & 30 & 30 & 10 \\
\hline Parent material (Matična podlaga) & & $\mathrm{R}$ & $\mathrm{R}$ & $\mathrm{R}$ & $\mathrm{R}$ & RA \\
\hline Soil (Tla) & & $\mathrm{Eu}$ & Dy & Dy & Dy & Dy \\
\hline Stoniness in \% (Kamnitost v \%) & & 10 & 1 & 10 & 1 & 5 \\
\hline Cover of tree layer in \% (Zastiranje drevesne plasti v \%) & E3 & 80 & 80 & 80 & 80 & 80 \\
\hline Cover of shrub layer in \% (Zastiranje grmovne plasti v \%): & E2 & 5 & 20 & 20 & 30 & 10 \\
\hline Cover of herb layer in \% (Zastiranje zeliščne plasti v \%): & E1 & 80 & 90 & 90 & 90 & 60 \\
\hline Cover of moss layer in \% (Zastiranje mahovne plasti v \%) & E0 & . & 5 & 1 & 10 & 1 \\
\hline Maximum diameter of trees (Največji prsni premer dreves) & $\mathrm{cm}$ & 20 & 35 & 30 & 25 & 30 \\
\hline Maximum height of tress (Največja drevesna višina) & $\mathrm{m}$ & 10 & 18 & 15 & 12 & 10 \\
\hline Number of species (Število vrst) & & 25 & 37 & 29 & 25 & 25 \\
\hline Relevé area (Velikost popisne ploskve) & $\mathrm{m}^{2}$ & 200 & 400 & 400 & 200 & 200 \\
\hline Date of taking relevé (Datum popisa) & & $6 / 15 / 2012$ & $6 / 15 / 2012$ & $6 / 15 / 2012$ & $6 / 15 / 2012$ & $5 / 10 / 2015$ \\
\hline Quadrant (Kvadrant) & & $9848 / 2$ & $9848 / 2$ & $9848 / 2$ & $9848 / 2$ & $9748 / 4$ \\
\hline Coordinate GK Y (D-48) & $\mathrm{m}$ & 407895 & 408424 & 407975 & 408123 & 406498 \\
\hline Coordinate GK X (D-48) & $\mathrm{m}$ & 5116376 & 5116175 & 5116548 & 5116451 & 5119246 \\
\hline
\end{tabular}

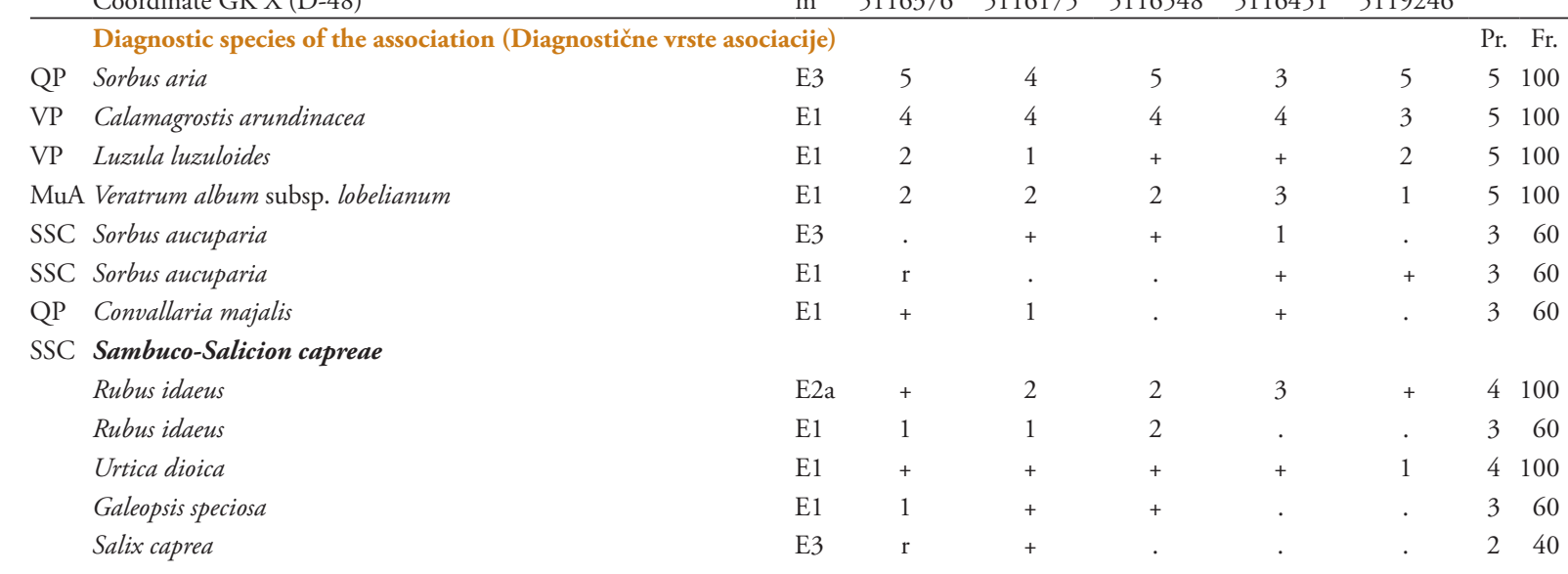


Number of relevé (Zaporedna številka popisa)

\section{2}

RP Rhamno-Prunetea

Rosa glauca

Rosa canina

QP Quercetalia pubescenti-petraeae

Melittis melissophyllum

Tanacetum corymbosum

$\mathrm{QR}$ Quercetalia roboris

Betula pendula

Betonica officinalis

TA Tilio-Acerion

Acer pseudoplatanus

Acer pseudoplatanus

Acer pseudoplatanus

Acer pseudoplatanus

Tilia platyphyllos

Thalictrum aquilegiifolium

Dryopteris affinis

Lamium orvala

Adoxa moschatellina

FS Fagetalia sylvaticae

Lilium martagon

Fraxinus excelsior

Dryopteris filix-mas

Symphytum tuberosum

Fagus sylvatica

Fagus sylvatica

Fagus sylvatica

Scrophularia nodosa

Epilobium montanum

Paris quadrifolia

Cardamine bulbifera

QF Querco-Fagetae

Anemone nemorosa

Ornithogalum pyrenaicum

Pyrus pyraster

Stellaria holostea

Erico-Pinetea

Molinia caerulea subsp. arundinacea

VP

Picea abies

Picea abies

Picea abies

Gentiana asclepiadea

Dryopteris dilatata

Maianthemum bifolium

Phegopteris connectilis

Oxalis acetosella

Larix decidua

Rosa pendulina

Vaccinium myrtillus

TG Trifolio-Geranietea

Verbascum lanatum

Valeriana collina (V. wallrothii)
E2a

E2b

E1

E1

E3

E1

E3

E2b

E2a

E1

E3b

E1

E1

E1

E1

E1

E2a

E1

E1

E3

E2

E

E

E1

E1

E1

E1

E1

E2b

E1

E1

E3

E2a

E1

E1

E1

E1

E1

E1

E3b

E1

E1

E1
120

120

120

120

240

120

360

20

360

480

120

120

120

120

$\begin{array}{ll}1 & 20 \\ 1 & 20\end{array}$

480

240

240

120

120

120

240

120

120

120

120

5100

120

120

120

120

$4 \quad 80$

120

120

360

360

360

240

240

120

120

120

240

E1 


\begin{tabular}{|c|c|c|c|c|c|c|c|c|c|}
\hline & Number of relevé (Zaporedna številka popisa) & & 1 & 2 & 3 & 4 & 5 & Pr. & Fr. \\
\hline \multicolumn{10}{|c|}{ MuA Mulgedio-Aconitetea } \\
\hline & Athyrium filix-femina & E1 & 1 & 1 & 2 & 2 & 1 & 5 & 100 \\
\hline & Senecio ovatus & E1 & 1 & 2 & + & 1 & + & 5 & 100 \\
\hline & Polygonatum verticillatum & E1 & + & . & + & 1 & . & 3 & 60 \\
\hline & Aconitum lycoctonum s. lat. & E1 & . & + & . & $\mathrm{r}$ & . & 2 & 40 \\
\hline & Stellaria nemorum agg. & E1 & . & . & 1 & . & + & 2 & 40 \\
\hline & Geranium sylvaticum & E1 & + & . & . & . & . & 1 & 20 \\
\hline & Salix appendiculata & E2 & . & . & . & . & + & 1 & 20 \\
\hline \multirow[t]{3}{*}{$\mathrm{FB}$} & Festuco-Brometea, Elyno-Seslerietea & & & & & & & & \\
\hline & Cirsium erisithales & E1 & $\mathrm{r}$ & + & . & . & . & 2 & 40 \\
\hline & Iris sibirica subsp. erirrhiza & E1 & $\mathrm{r}$ & . & . & . & . & 1 & 20 \\
\hline ES & Betonica alopecuros & E1 & . & + & . & . & . & 1 & 20 \\
\hline \multirow[t]{2}{*}{$\mathrm{CU}$} & Calluno-Ulicetea & & & & & & & & \\
\hline & Carex pallescens & E1 & . & + & . & $\mathrm{r}$ & . & 2 & 40 \\
\hline \multirow[t]{6}{*}{ M } & Mosses (Mahovi) & & & & & & & & \\
\hline & Atrichum undulatum & E0 & + & 1 & + & 1 & . & 4 & 80 \\
\hline & Hypnum cupressiforme & E0 & . & . & + & . & + & 2 & 40 \\
\hline & Polytrichum formosum & E0 & . & . & . & + & . & 1 & 20 \\
\hline & Ctenidium molluscum & E0 & . & . & . & . & 1 & 1 & 20 \\
\hline & Isothecium alopecuroides & E0 & . & . & . & . & + & 1 & 20 \\
\hline
\end{tabular}

\section{Legend - Legenada}

$\mathrm{R}$ Chert-roženec

A Limestone-apnenec

Eu Eutryc brown soil - evtrična rjava tla

Dy Dystric brown soil - distrična rjava tla 